\title{
Hyperscaling in the Broken Symmetry Phase of Dyson's Hierarchical Model
}

\author{
J. J. Godina \\ Dept. de Fisica, CINVESTAV-IPN , Ap. Post. 14-740, Mexico, D.F. 07000 \\ Y. Meurice and M. B. Oktay \\ Dept. of Physics and Astr., Univ. of Iowa, Iowa City, Iowa 52242, USA
}

\begin{abstract}
We use polynomial truncations of the Fourier transform of the local measure to calculate the connected q-point functions of Dyson's hierarchical model in the broken symmetry phase. We show that accurate values of the connected 1, 2 and 3 point functions can be obtained at large volume and in a limited range of constant external field coupled linearly to the field variable. We introduce a new method to obtain the correct infinite volume and zero external field extrapolations. We extract the leading critical exponents and show that they obey the scaling and hyperscaling relations with an accuracy ranging from $10^{-5}$ to $5 \times 10^{-3}$. We briefly discuss how to improve the method of calculation.
\end{abstract}




\section{INTRODUCTION}

Spontaneous symmetry breaking plays a fundamental role in our understanding of the mass generation mechanism of the elementary particles. One of the simplest field theory model where it is observed is scalar theory. Despite its simplicity, there exists no known analytical method which allows one to elucidate quantitatively all the dynamical questions which can be asked about scalar field theory in various dimensions. From a sample of the recent literature on scalar field theory, one can see that the Monte Carlo method is a popular tool to settle questions such as the existence of non-perturbative states [1], large rescaling of the scalar condensate [2] or Goldstone mode effects [3].

The Monte Carlo method allows us to approach quantum field theory problems for which there are no known reliable series expansions. The main limitations of the method are the size of the lattice which can be reached and the fact that the errors usually decrease like $t^{-1 / 2}$, where $t$ is the CPU time used for the calculation. If, in the next decades, a better knowledge of the fundamental laws of physics has to rely more and more on precision tests, one should complement Monte Carlo methods with new computational tools which emphasize numerical accuracy.

This motivated us to use "hierarchical approximations" as a starting point, since they allow a more easy use of the renormalization group $(\mathrm{RG})$ transformation. Examples of hierarchical approximations are Wilson's approximate recursion formula [4] or the hierarchical model [5]. In the symmetric phase, we have found [6] that polynomial truncations of the Fourier transform of the local measure provide spectacular numerical accuracy, namely, various types of errors decrease like $\mathrm{e}^{-A t^{u}}$, for some positive constant $A$ of order 1 when $t$ is measured in minutes of CPU time and $0.5 \leq u \leq 1$. In particular, $t$ only grows as the logarithm of the number of sites $L^{D}$ and the finite-size effects decay like $L^{-2}$ when $L$ (the linear size) becomes larger than the correlation length. This method of polynomial truncations was used [7] to calculate the critical exponent $\gamma$ in the symmetric phase for the hierarchical model with estimated errors of the order of $10^{-12}$. The result was confirmed by calculating 
the largest eigenvalue of the linearized $R G$ about the accurately determined non-trivial fixed point [8].

Thanks to the polynomial approximation, very accurate information can be encoded in a very small set of numbers. In the symmetric phase, this approximation is numerically stable when the number of sites becomes arbitrarily large and the high-temperature fixed point is reached. On the other hand, in the broken symmetry phase, numerical instabilities appear after a certain number of iterations following the bifurcation, and it is not possible to completely get rid of the finite size effects with the straightforward procedure used in the symmetric phase. This issue was briefly discussed in section III.E of Ref. [7].

In this paper, we analyze the numerical instabilities of the low-temperature phase in a quantitative way. We show that in spite of these numerical instabilities, it is possible to take advantage of the iterations for which the low-temperature scaling is observed to obtain reliable extrapolations of the magnetization, first to infinite volume at non-zero external field and then to zero external field. We then present a more pratical method of extrapolation which we apply to calculate the connected $q$-point functions at zero momentum $G_{q}^{c}(0)$ for $q=1,2$ and 3. Finally, we use these calculations to extract the leading critical exponents and we check the hyperscaling relations among these exponents.

The paper is organized as follows. In section II we show how to construct recursively the generating function for the $G_{q}^{c}(0)$ when a magnetic field is introduced. In section III, we review the scaling and hyperscaling relations among the critical exponents and explain how they should be understood in the case of the hierarchical model. Hyperscaling [9] usually refers to scaling relations involving the dimension explicitly. Dyson's hierarchical model has no intrinsic dimensionality but rather a continuous free parameter usually denoted by $c$ introduced in section II, which controls the decay of the interactions among blocks of increasing sizes. This parameter can be tuned in order to insure that a massless field has scaling properties that can be compared with those of nearest neighbor models in $D$ dimensions. In the past we have chosen the parametrization $c=2^{1-2 / D}$, however this is not the only possible one. In section III. C, we show that a more general parametrization of $c$, 
(which includes $\eta$ ) combined with linear arguments yields predictions that are identical to the conventional predictions obtained from scaling and hyperscaling. We want to emphasize that the main prediction of the linear theory - that can be interpreted as a hyperscaling relation - can be expressed in terms of $c$ only and is given in general by Eq. (3.15). For $c=2^{1 / 3}$, this general equation together with the accurate result of Ref. [7] implies

$$
\gamma_{q}=1.29914073 \ldots \times(5 q-6) / 4
$$

where $\gamma_{q}$ is the leading exponent corresponding to the connected $q$-point function.

We then proceed to verify the predictions of Eq. (1.1) by doing actual calculations at various values of the inverse temperature $\beta$ near criticality. This is a rather challenging task because as one moves away from the unstable fixed point, in the low-temperature side, rapid oscillations appear in the Fourier transform of the local measure and the polynomial approximation ultimately breaks down. This is the cause of the numerical instabilities mentioned above. As a consequence, a relatively small number of iterations can be performed with a reasonable accuracy in the low-temperature phase. This is explained in section IV where we also show that the number of numerically accurate iterations in the low-temperature phase scales like the logarithm of the degree of the polynomial. For the calculations discussed later in the paper, we have used a polynomial truncation of order 200 . With this choice, the number of iterations where an approximate low-temperature scaling is observed is slightly larger than 10. Since for Dyson's hierarchical model the number of sites is halved after each iteration, it means roughly speaking that in correlation length units we can only reach volumes which are $2^{10} \simeq 10^{3}$. If we use the $D=3$ interpretation of $c=2^{1 / 3}$, this means that the linear size, denoted by $L$, which can be reached safely are at most 10 times the correlation lengths.

Despite this limitation, the magnetization reaches its infinite volume limit with clearly identifiable $L^{-2}$ corrections provided that the external magnetic field is not too large (otherwise the polynomial approximation breaks down) or not too small (otherwise a linear analysis applies and there is no spontaneous magnetization). The exact intermediate range 
of the magnetic field for which the connected $q$-point functions reach an infinite volume limit with the characteristic $L^{-2}$ corrections is discussed in section V. In this intermediate range, two methods of extrapolation can be used. The first is the standard one which consists in extrapolating to infinite volume at fixed external field and then to zero external field. On the other hand, within the intermediate range of magnetic field mentioned above, the magnetization at finite volume can be fitted very accurately with a straight line which provides an extrapolation to zero magnetic field. This extrapolation has no physical meaning but it also reaches an infinite volume limit with $L^{-2}$ corrections when the volume increases. This limit coincides with an accuracy of 6 significant figure with the limit obtained with the first method; in other words within the estimated errors of the calculation. The second procedure is much more practical because it does not require any overlap among the acceptable regions of magnetic field when the volume increases. The second method will be used to calculate the higher point functions.

Proceeding this way, we calculate the connected $q$-point functions at zero momentum $G_{q}^{c}(0)$, for $q=1,2$ and 3 and for various values of the inverse temperature $\beta$. The results are reported in section VI. The critical exponents are then estimated by using a method discussed in Ref. [7] where we selected a region of $\beta$ for which the combined effects of the errors due to subleading corrections and the numerical round-off could be minimized. Using linear fits within this limited range of $\beta$, we found exponents in agreement with the prediction of hyperscaling given in Eq. (3.15) with an accuracy of $10^{-5}$ for the magnetization, $4 \times 10^{-5}$ for the susceptibility and $5 \times 10^{-3}$ for the 3 -point function. As far as the first two results are concerned, the accuracy compares well with the accuracy that can usually be reached with a series analysis or the Monte Carlo method. Nevertheless, there is room for improvement: one should be able to "factor out" the rapid oscillations in the Fourier transform of the local measure and treat them exactly. This is discussed briefly in the conclusions. 


\section{INTRODUCTION OF A MAGNETIC FIELD}

In this section, we describe Dyson's Hierarchical Model [5.10] coupled to a constant magnetic field. All calculations are performed at large but finite volume. The total number of sites denoted $2^{n_{\max }}$. We label the sites with $n_{\max }$ indices $x_{n_{\max }}, \ldots, x_{1}$, each index being 0 or 1 . In order to visualize this notation, one can divide the $2^{n_{\max }}$ sites into two blocks, each containing $2^{n_{\max }-1}$ sites. If $x_{n_{\max }}=0$, the site is in the first box, if $x_{n_{\max }}=1$, the site is in the second box. Repeating this procedure $n_{\max }$ times (for the two boxes, their respective two sub-boxes, etc.), we obtain an unambiguous labeling for each of the sites.

The non-local part of the action (i.e. the "kinetic term") of Dyson's Hierarchical model reads

$$
S_{k i n}=-\frac{\beta}{2} \sum_{n=1}^{n_{\max }}\left(\frac{c}{4}\right)^{n} \sum_{x_{n_{\max }, \ldots, x_{n+1}}}\left(\sum_{x_{n}, \ldots, x_{1}} \phi_{\left(x_{\left.n_{\max }, \ldots, x_{1}\right)}\right)^{2}} .\right.
$$

The index $n$, referred to as the 'level of interaction' hereafter, corresponds to the interaction of the total field in blocks of size $2^{n}$. The constant $c$ is a free parameter which describes the way the non-local interactions decay with the size of the blocks. We often use the parametrization

$$
c=2^{1-2 / D}
$$

in order to approximate $D$-dimensional models. This question will be discussed later (see Eq. (3.19) for a generalization of Eq. (2.2)).

A constant external source $H$, called "the magnetic field" later, is coupled to the total field. This can be represented by an additional term in the action

$$
S_{H}=-H \sum_{x_{n_{\max }, \ldots, x_{1}}} \phi_{\left(x_{\left.n_{\max }, \ldots, x_{1}\right)}\right.}
$$

However due to the linearity of the coupling, $\mathrm{e}^{-S_{H}}$ factorizes into local pieces and this

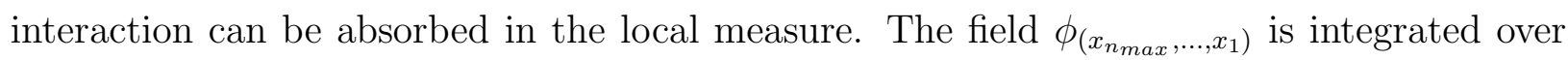
with a local measure 


$$
W_{0}(\phi, H) \propto W_{0}(\phi) \mathrm{e}^{H \phi}
$$

where $W_{0}(\phi)$ is the local measure at zero magnetic field. For simplicity, we use the convention that if the magnetic field does not appear explicitly in an expression (e. g. , $\left.W_{0}(\phi)\right)$ the quantity should be understood at zero magnetic field. The constant of proportionality refers to the fact that we require both $W_{0}(\phi, H)$ and $W_{0}(\phi)$ to be normalized as probability distributions. Since we are interested in universal properties, we will use a single local measure, namely the Ising measure, $W_{0}(\phi)=\delta\left(\phi^{2}-1\right)$. Numerical experiments in Ref. [7] show that the universal properties are very robust under changes in the local measure.

At $H=0$, the recursion relation corresponding to the integration of the fields in boxes of size 2, keeping the sum of the 2 fields in each box constant reads

$$
W_{n+1}(\phi)=\frac{C_{n+1}}{2} e^{\beta / 2(c / 4)^{n+1} \phi^{2}} \int d \phi^{\prime} W_{n}\left(\frac{\left(\phi-\phi^{\prime}\right)}{2}\right) W_{n}\left(\frac{\left(\phi+\phi^{\prime}\right)}{2}\right),
$$

where $C_{n+1}$ is a normalization factor which will be fixed in order to obtain a probability distribution. Introducing the Fourier representation as in Refs. [6,7]

$$
W_{n}(\phi)=\int \frac{d k}{2 \pi} e^{i k \phi} \hat{W}_{n}(k)
$$

and a rescaling of the "source" $k$ by a factor $(c / 4)^{1 / 2}$ at each iteration ,

$$
R_{n}(k)=\hat{W}_{n}\left(k(c / 4)^{n / 2}\right)
$$

the recursion relation becomes

$$
R_{n+1}(k)=C_{n+1} \exp \left(-\frac{1}{2} \beta \frac{\partial^{2}}{\partial k^{2}}\right)\left(R_{n}\left(k(c / 4)^{1 / 2}\right)\right)^{2}
$$

We fix the normalization constant $C_{n}$ is such way that $R_{n}(0)=1 . R_{n}(k)$ has then a direct probabilistic interpretation. If we call $M_{n}$ the total field $\sum \phi_{x}$ inside blocks of side $2^{n}$ and $\langle\ldots\rangle_{n}$ the average calculated without taking into account the interactions of level strictly larger than $n$, we can write (at $H=0$ )

$$
R_{n}(k)=\sum_{q=0}^{\infty} \frac{(-i k)^{2 q}}{(2 q) !}\left\langle\left(M_{n}\right)^{2 q}\right\rangle_{n}(c / 4)^{q n}
$$


The introduction of the magnetic field is a very simple operation. The basic equation reads

$$
W_{n}(\phi, H) \propto W_{n}(\phi) \mathrm{e}^{H \phi}
$$

This can be seen in many different ways. One of them is to use Eq. (2.5) and realize that the $\phi^{\prime}$ drops out of the magnetic interactions. Another one consists in realizing that due to the linearity, one can split Eq. (2.3) into sum over boxes of any desired size. In Fourier transform, this implies that

$$
\hat{W}_{n}(k, H) \propto \hat{W}_{n}(k+i H)
$$

The normalization factor is fixed by the condition $\hat{W}_{n}(0, H)=1$ which guarantees that $W_{n}(\phi, H)$ is a probability distribution and that $\hat{W}_{n}(k, H)$ generates the average values of the positive powers of the total field. More explicitly,

$$
\begin{aligned}
\hat{W}_{n}(k, H) & =\frac{\hat{W}_{n}(k+i H)}{\hat{W}_{n}(i H)} \\
& =\sum_{q=0}^{\infty} \frac{(-i k)^{q}}{q !}\left\langle\left(M_{n}\right)^{q}\right\rangle_{n, H} .
\end{aligned}
$$

From a conceptual point of view, as well as from a practical one, it is easier to deal with the rescaled quantity $R_{n}(k)$. Near the fixed point of Eq. (2.8), we have the approximate behavior

$$
\left\langle\left(M_{n}\right)^{2 q}\right\rangle_{n} \propto(4 / c)^{q n}
$$

In terms of the rescaled function, we can rewrite Eq. (2.13) as

$$
\frac{R_{n}\left(k+i H(4 / c)^{n / 2}\right)}{R_{n}\left(i H(4 / c)^{n / 2}\right)}=\sum_{q=0}^{\infty} \frac{(-i k)^{q}}{q !}\left\langle\left(M_{n}\right)^{q}\right\rangle_{n, H}(c / 4)^{q n / 2} .
$$

The connected Green's functions can be obtained by taking the logarithm of this generating function. 


\section{ABOUT HYPERSCALING}

\section{A. General Expectations}

The main numerical results obtained in this paper are the calculations of the critical exponents corresponding to the singularity of the connected $q$-point functions for $q=1,2$ and 3. For definitiness we use the notation

$$
G_{q}^{c}(0) \propto\left(\beta-\beta_{c}\right)^{-\gamma_{q}}
$$

for the leading singularities in the low-temperature phase. We assume that the reader is familiar with the commonly used notations [11] for the critical exponents. For $q=1$, we have $\gamma_{1}=-\beta$ which should not be confused with the inverse temperature. After this subsection, we keep using the notation $\beta$ for the inverse temperature. For $q=2$, we have $\gamma_{2}=\gamma^{\prime}$. If one assumes that the scaled magnetization $M /\left(T-T_{c}\right)^{\beta}$ is a function of the scaled magnetic field $H /\left(T-T_{c}\right)^{\Delta}$ only, one obtains that

$$
\gamma_{q+1}-\gamma_{q}=\Delta
$$

for any $q$. The exponent $\Delta$ is often called the gap exponent and should not be confused with the exponent associated with the subleading corrections to the scaling laws.

In general, there exists 7 relations among the 10 critical exponents $\alpha, \alpha^{\prime}, \beta, \gamma, \gamma^{\prime}$, $\Delta, \delta, \nu, \nu^{\prime}$, and $\eta$, in which the dimension of the system does not enter explicitly. These are the so-called scaling relations [11] which stimulated the development of the RG method. Their explicit form is

$$
\begin{aligned}
& \alpha=\alpha^{\prime} \\
& \gamma=\gamma^{\prime} \\
& \nu=\nu^{\prime} \\
& \alpha+2 \beta+\gamma=2 \\
& \Delta=\beta+\gamma
\end{aligned}
$$




$$
\begin{aligned}
& \Delta=\beta \delta \\
& \gamma=(2-\eta) \nu .
\end{aligned}
$$

Eq. (3.7) can be seen as an obvious version of Eq. (3.2) for $q=1$, but has also a non-trivial content summarizing Eq. (3.2) for all the higher $q$.

In addition there exists one relation where the dimension enters explicitly, for instance:

$$
D \nu=2-\alpha .
$$

Other relations may be obtained by combining Eq. (3.10) with the scaling relations. Proceeding this way, we obtain a relation of relevance for the rest of the discussion, namely

$$
\beta=\frac{(D-2+\eta)}{2(2-\eta)} \gamma
$$

The relations involving the dimension explicitly are usually called hyperscaling relations [12]. A mechanism leading to a possible violation of hyperscaling (dangerous irrelevant variables) is explained in appendix D of Ref. [9]. If the 8 relations hold, we are left with only two independents exponents, for instance $\gamma$ and $\eta$.

Combining the hyperscaling relation (3.11) and the scaling relations (3.4) and (3.7), we obtain

$$
\begin{aligned}
\gamma_{q} & =\gamma+(q-2) \Delta \\
& =\gamma[-2 D+q(D+2-\eta)] /(4-2 \eta)
\end{aligned}
$$

\section{B. The Hierarchical Model (HT case)}

In the case of the hierarchical model, the exponents $\gamma_{q}$ of the high-temperature (HT) phase (so for $q$ even) can be estimated by using the linearized RG transformation. Since this subsection is the only part of this article where we will consider the high-temperature phase, we have not found useful to introduce special notations for $\gamma_{q}$ in this phase. When $\beta_{c}-\beta$ is 
small, the linearized RG transformation can be used for approximately $n^{\star}$ iterations, with $n^{\star}$ defined by the relation

$$
\left|\beta-\beta_{c}\right| \lambda^{n^{\star}}=1
$$

where $\lambda$ is the largest eigenvalue of the linearized RG transformation. After the transient behavior has died off and until $n$ reaches the value $n^{\star}$, we are near the fixed point and $R_{n}(k)$ does not change appreciably. Remembering that the field is rescaled by a factor $(c / 4)^{\frac{1}{2}}$ at each iteration (see Eq. (2.8)), we obtain the order of magnitude estimate for $G_{q}^{c}(0)$ after $n^{\star}$ iterations:

$$
G_{q}^{c}(0) \approx 2^{-n^{\star}}(4 / c)^{q n^{\star} / 2}
$$

For $n$ larger than $n^{\star}$, the non-linear effects become important. The actual value of $G_{q}^{c}(0)$ may still change by as much as 100 percent, however the order of magnitude estimate of Eq. (3.14) remains valid. This transition has been studied in detail in Ref. [13 in a simplified version of the model. Eliminating $n^{\star}$ in terms of $\beta_{c}-\beta$, we obtain the value of the leading exponents

$$
\gamma_{q}=\gamma[(q / 2) \ln (4 / c)-\ln 2] / \ln (2 / c)
$$

with

$$
\gamma=\ln (2 / c) / \ln \lambda
$$

This relationship has been successfully tested [6] in the symmetric phase for $q=4$ and $4 / c=2^{5 / 3}$.

\section{About Dimensionality}

We will now show that Eq. (3.15) is compatible with the general relation of Eq. (3.12) provided that we relate $c$ to a parameter $D$ which can be interpreted as the dimension of 
a nearest neighbor model approximated by the hierarchical model. We introduce a linear dimension $L$ such that the volume $L^{D}$ is proportional to the total number of sites $2^{n}$. From

$$
L \propto 2^{n / D}
$$

we can in general relate $c$ and $D$ by assuming a scaling of the total field

$$
\left\langle M_{n}^{2 q}\right\rangle_{n} \propto L^{(D+2-\eta) q}
$$

From comparison with Eq. 2.13) this would imply that

$$
(4 / c)=2^{(D+2-\eta) / D} .
$$

Substituting in Eq. (3.15), we reobtain the general Eq. (3.12).

Since in the infinite volume limit, the kinetic term is invariant under a RG transformation, we have chosen in the past to use Eq. (3.19) with $\eta=0$. This is our conventional definition of $c$ given in Eq. (2.2). This is the same as saying that when we are near the fixed point, the total field in a box containing $2^{n}$ sites, scales with the number of sites in the same way as a massless Gaussian field. This obviously implies that in the vicinity of a Gaussian fixed point the total field scales exactly like a massless Gaussian field in $D$ dimension. On the other hand, an interacting massless field will also scale like a free one, which is not a bad approximation in $D=3$. This is an unavoidable feature which will need to be corrected when one tries to improve the hierarchical approximation.

We emphasize that this interpretation has no bearing on the validity of the calculations performed. What matters in our calculation is the value of $4 / c$. In the following, we have used $4 / c=2^{5 / 3}$, which can be interpreted either as $D=3$ and $\eta=0$ or, for instance, as $D=2.97$ and $\eta=0.02$.

\section{The Low-Temperature Case}

The extension of the argument for odd and even values of $q$ in the broken symmetry phase is somehow non-trivial. Since we need to take the infinite volume limit before taking 
the limit of a zero magnetic field, we need some understanding of the non-linear behavior. Some aspects of the non-linear behavior are discussed in section $\nabla$. In the following, we will show numerically that Eq. (3.15) holds in good approximation in the broken symmetry phase for $4 / c=2^{5 / 3}$. With this choice of $4 / c$ and the corresponding value of $\gamma$ calculated in Ref. [7], Eq. (3.15) implies Eq. (11.1) given in the introduction. The verification of this relation for $q=1,2$ and 3 is the main numerical result discussed in the following chapters.

\section{POLYNOMIAL TRUNCATIONS}

In the following we will exclusively consider the case of an Ising measure

$$
R_{0}(k)=\cos (k)
$$

This restriction is motivated by accurate checks [7] of universality based on calculations with other measures. Given that $R_{0}$ can be expanded into a finite number of eigenfunctions of $\exp \left(-\frac{1}{2} \beta \frac{\partial^{2}}{\partial k^{2}}\right)$, one can in principle obtain exact expressions for the next $R_{n}(k)$, for instance

$$
R_{1}(k)=\frac{1+\mathrm{e}^{\beta c / 2} \cos (k \sqrt{c})}{1+\mathrm{e}^{\beta c / 2}} .
$$

One can in principle repeat this procedure. At each iteration, one obtains a superposition of cosines of various frequencies. For a given numerical value of $c, n$ iterations of this exact procedure requires to store $2^{n-1}+1$ numerical coefficients. The memory size thus scales like $2^{n}$, while the CPU time scales like $4^{n}$. If $\beta$ differs from $\beta_{c}$ by $10^{-10}$, one needs at least 80 iterations in order to eliminate the finite-size effects. Such a calculation using the exact method can be ruled out by practical considerations.

We will thus try to extend the approximate methods that we have used successfully in the symmetric phase [7], where the function $R_{n}(k)$ was calculated using finite dimensional approximations [6] of degree $l_{\max }$ :

$$
R_{n}(k)=1+a_{n, 1} k^{2}+a_{n, 2} k^{4}+\ldots+a_{n, l_{\max }} k^{2 l_{\max }} .
$$


After each iteration, non-zero coefficients of higher order $\left(a_{n+1, l_{\max +1}}\right.$ etc. $)$ are obtained, but not taken into account as a part of the approximation in the next iteration. The recursion formula for the $a_{n, m}$ reads [6] :

$$
a_{n+1, m}=\frac{\sum_{l=m}^{l_{\max }}\left(\sum_{p+q=l} a_{n, p} a_{n, q}\right)[(2 l) ! /(l-m) !(2 m) !](c / 4)^{l}[-(1 / 2) \beta]^{l-m}}{\sum_{l=0}^{l_{\max }}\left(\sum_{p+q=l} a_{n, p} a_{n, q}\right)[(2 l) ! / l !](c / 4)^{l}[-(1 / 2) \beta]^{l}} .
$$

The method to identify $\beta_{c}$ has been discussed in detail in Ref. [6] and consists in finding the bifurcation in the ratio $a_{n+1,1} / a_{n, 1}$. In the following, we simply call this quantity "the ratio". If $\beta<\beta_{c}$, the ratio drops to $c / 2$ for $n$ large enough. In this case, the numerical stability of the infinite volume limit is excellent and allows extremely accurate determination of the renormalized quantities. If $\beta>\beta_{c}$, the ratio "jumps" suddenly a few iterations after $n^{\star}$ is reached and stabilizes near the value $c$, corresponding to the low-temperature scaling. This is seen from Eq. (2.13). Since $\left\langle M_{n}^{2}\right\rangle_{n}$ grows like $4^{n}$, as one expects in the low-temperature phase, and remembering that there is a rescaling of $c / 4$ at each iteration, the coefficient of $k^{2}$ grows like $c^{n}$. This implies a ratio equal to $c$. In our calculation, $c=1.25992 \ldots$. Unfortunately, the number of iterations where the low-temperature scaling is observed is rather small. Subsequently, the ratio drops back to 1. As we shall explain at length, this is an effect of the polynomial truncation. The length of the "shoulder" were the low-temperature scaling is observed increases if we increase $l_{\text {max }}$. This situation is illustrated in Figure 1. 


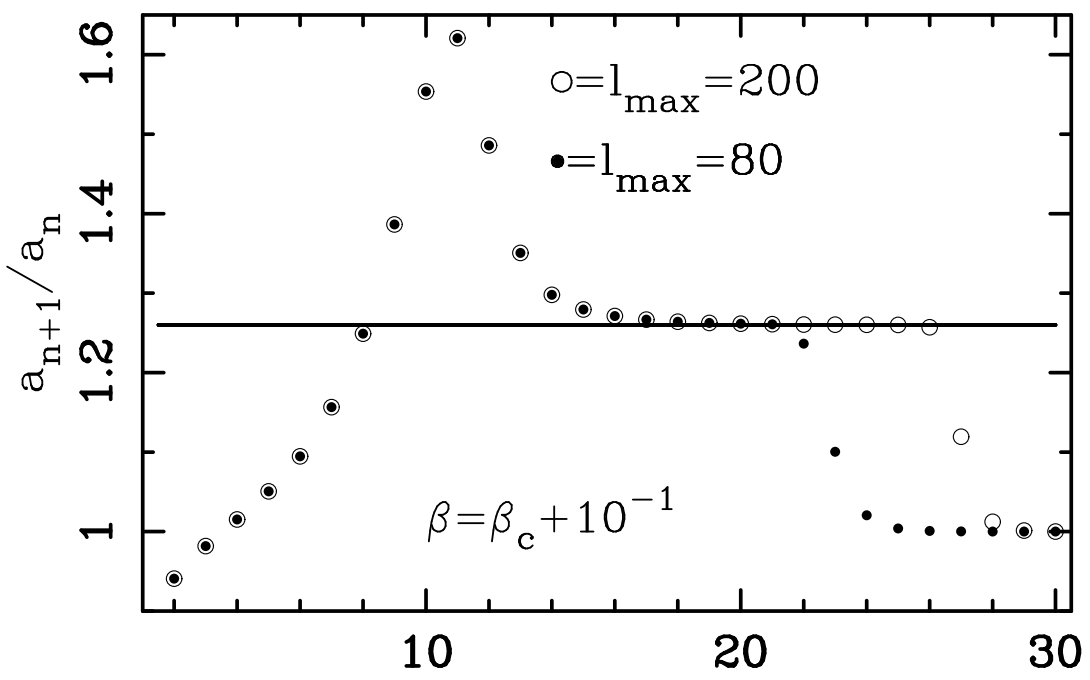

FIG. 1. The low-temperature shoulder at $\beta=\beta_{c}+10^{-1}$ for $l_{\max }=200$ (empty circles) and $l_{\max }=80$ (filled circles) as a function of $l_{\max }$.

No matter how large $l_{\max }$ is, for $n$ large enough, the ratio eventually drops back to 1 . This reflects the existence of a stable fixed point for the truncated recursion formula. The values $a_{l}^{\star}$ of $a_{l}$ at this fixed point for various $l_{\max }$ are shown in Figure 2 .

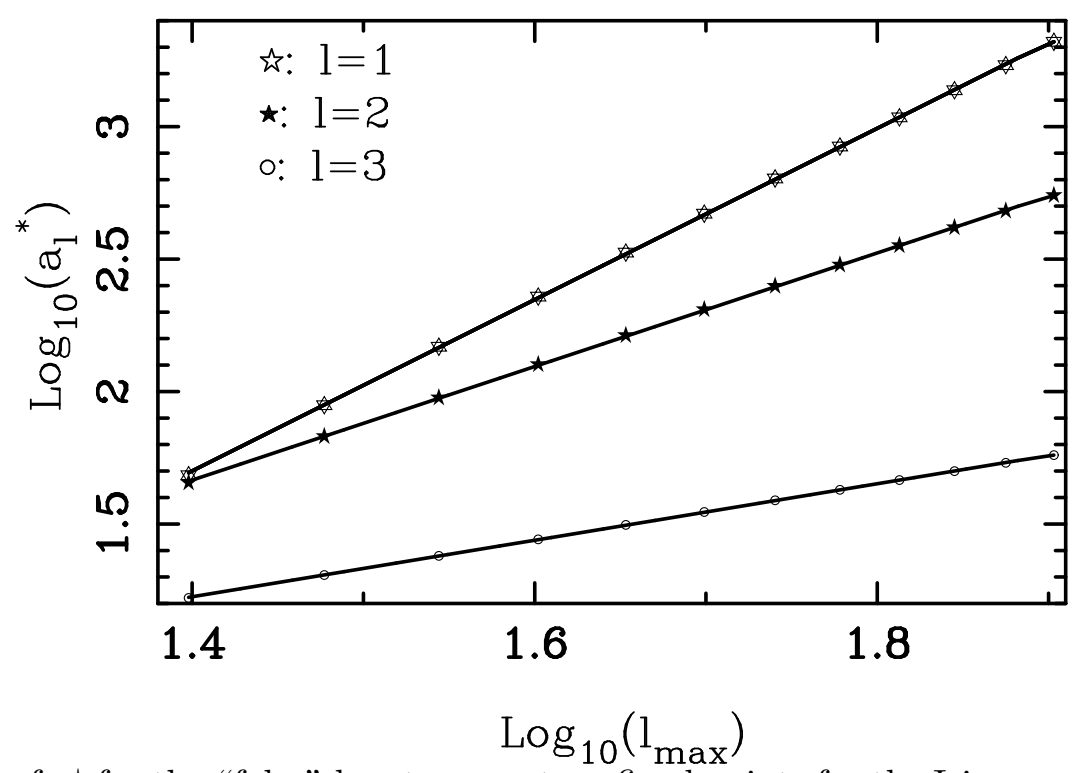

FIG. 2. Value of $a_{l}^{\star}$ for the "false" low-temperature fixed points for the Ising case in 3 dimensions for $l=1$ (circles), $l=2$ (filled stars), $l=3$ (empty stars)

We see a clear evidence for a dependence of the form 


$$
a_{l}^{\star} \propto\left(l_{\max }\right)^{l} .
$$

This means that the stable fixed point is an effect of the polynomial truncations and has no counterpart in the original model.

It is possible to evaluate the value of $n$ for which the low-temperature shoulder ends. A detailed study shows that for $n$ large enough, we have in good approximation

$$
R_{n}(k) \simeq \cos \left(\mathcal{M} c^{n / 2} k\right),
$$

where $\mathcal{M}$ is the magnetization density in the infinite volume limit. If we assume that $R_{n}(k)$ is exactly as in Eq. (4.6), then we can use the basic recursion formula (2.8) in order to obtain the corresponding $R_{n+1}(k)$. Using $2 \times \cos ^{2}(x)=1+\cos (2 x)$, we can reexpress $\left(R_{n}\left(k(c / 4)^{1 / 2}\right)\right)^{2}$ as a superposition of eigenfunctions of the one-dimensional Laplacian. When the exponential of the Laplacian in Eq. (2.8) acts on the non-constant modes it becomes $\exp \left(\beta \mathcal{M}^{2} c^{n+1} / 2\right)$. In the polynomial truncation of the recursion relation, this exponential is replaced by $l_{\max }$ terms of its Taylor expansion. This approximation is valid if the argument of the exponential is much smaller than $l_{\max }$. Consequently, we obtain that the polynomial truncation certainly breaks down if $n$ is larger than $n_{b}$ such that

$$
n_{b}+1 \simeq\left[\ln (2 / \beta)-\ln \left(\mathcal{M}^{2}\right)+\ln \left(l_{\max }\right)\right] / \ln c .
$$

If the estimate of Eq. (3.14) extends to the low-temperature phase, one realizes that the second term of (4.7) is roughly $n^{\star}$ while the third term stands for the length of the peak and the shoulder. Plugging the approximate values 1.1 for $\beta$ and 0.7 for $\mathcal{M}$ (see section $\bar{\nabla}$ ), we obtain $n_{b}=23$ for $l_{\max }=80$ and $n_{b}=27$ for $l_{\max }=200$. A quick glance at Figure [1], shows that these estimates coincide with the first drastic drops of the low-temperature shoulder.

One can in principle extend indefinitely the low-temperature shoulder by increasing $l_{\max }$. However, the CPU time $t$ necessary for $n$ iterations of a quadratic map in dimension $l_{\max }$ grows like

$$
t \propto n\left(l_{\max }\right)^{2}
$$


As we will show in section $\square$, the finite-size effects on $G_{q}^{c}(0)$ are of the order $(c / 2)^{n_{s}}$ where $n_{s}$ is the number of points on the shoulder. This behavior has been demonstrated [6] in the high-temperature phase and we will see later that it also applies in the low-temperature phase. From the previous discussion $n_{s} \approx \ln l_{\max } / \ln c$. This implies that the finite-size effects $\mathcal{E}$ are of the order

$$
\mathcal{E} \propto\left(l_{\text {max }}\right)^{\ln (c / 2) / \ln c}
$$

Using Eq. (4.8) and the value of $c$ expressed in terms of $D$ according to Eq. (3.19) with $\eta=0$, we obtain

$$
\mathcal{E} \propto t^{-1 /(D-2)}
$$

In particular, for the value $4 / c=2^{5 / 3}$ used hereafter, the errors decrease like $t^{-1}$. Consequently, we should try to modify the method in such a way that the rapidly oscillating part of $R_{n}(k)$ is treated without polynomial approximations. This possibility is presently under investigation. One can nevertheless obtain results with an accuracy competing with existing methods by using the finite data on the short shoulder in order to extrapolate to the infinite volume limit result. This procedure is made possible by the rather regular way the renormalized quantities approach this limit.

\section{THE EXTRAPOLATION TO INFINITE VOLUME}

\section{A. Preliminary Remarks}

There is no spontaneous magnetization at finite volume. This well-known statement can be understood directly from Eq. (2.14). As explained at the beginning of section IV, at finite $n, R_{n}(k)$ is simply a superposition of cosines with finite positive coefficients provided that $\beta$ is real. However if $\beta$ is complex, these coefficients have singularities. This comes from the normalization factor, needed when we impose the condition $R_{n}(0)=1$, which has zeroes in the complex plane. The behavior of these zeroes has been studied in Ref. [14] for $n$ 
between 6 and 12. As the volume increases, these zeroes "pinch" the critical point. However at finite $n$, there are no zeroes on the real axis. In conclusion, at real $\beta$ and finite $n, R_{n}(k)$ is an analytical function of $k$. For any given $n$, we can always take the magnetic field $H$ small enough in order to have

$$
\left|H(4 / c)^{n / 2}\right| \ll 1
$$

If we express $c$ in terms of the linear dimension using Eqs. (2.2) and (3.17) this translates into

$$
|H| \ll L^{-(D+2) / 2}
$$

Given the analyticity of $R_{n}(k)$, one can then use Eq. (2.14) in the linear approximation. In this limit,

$$
\left\langle M_{n}\right\rangle_{n} \simeq-2 a_{n, 1} H(4 / c)^{n}
$$

and the magnetization vanishes linearly with the magnetic field.

On the other hand, for any non-zero $H$, no matter how small its absolute value is, one can always find a $n$ large enough to have $\left|H(4 / c)^{n / 2}\right| \gg 1$. The non-linear effects are then important and Eq. (5.3) does not apply. In addition it is assumed (and will be verified explicitly later) that when such a $n$ are reached, the value of the $G_{q}^{c}(0)$ stabilizes at an exponential rate. One can then, first extrapolate at infinite volume for a given magnetic field, and then reduce the magnetic field in order to extrapolate a sequence of infinite volume limits with decreasing magnetic field, towards zero magnetic field. Again, this procedure requires some knowledge about the way the second limit in reached. In the case considered here (one scalar component), the limit is reached by linear extrapolation. In systems with more components, the Nambu-Goldstone modes create a square root behavior [15]

$$
M\left(T<T_{c}, H>0\right)=M\left(T, 0^{+}\right)+C H^{1 / 2} .
$$

which has been observed for $O(4)$ models using Monte Carlo simulations [3]. We now discuss the application of the procedure outlined above in the simplest case. 


\section{B. Calculation of the magnetization}

In this subsection we discuss the calculation of the infinite volume limit of the magnetization. The magnetization density at finite volume is defined as

$$
\mathcal{M}_{n}(H)=\frac{\left\langle M_{n}\right\rangle_{n, H}}{2^{n}}
$$

We call it "the magnetization" when no confusion is possible. For definiteness, we have chosen a special value $\beta=\beta_{c}+10^{-1}$ and calculated the magnetization by plugging numerical values of $H$ in Eq. (2.14) and expanding to first order in $k$. The results are shown in Fig. 3 for $n=17$ and $l_{\max }=200$.

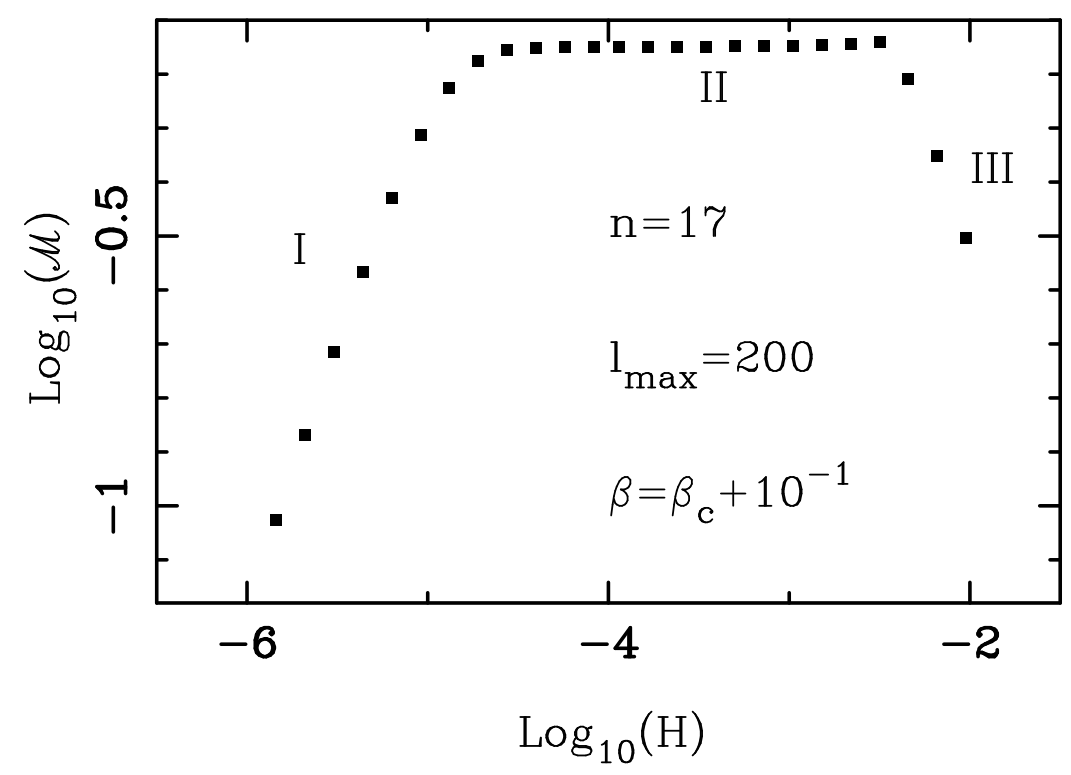

FIG. 3. $\log _{10}(\mathcal{M})$ versus $\log _{10}(H)$ at $n=17$ for $\beta=\beta_{c}+10^{-1}$.

As one can see, we have three different regions. The first one (I) is the region where the linear approximation described above applies. For the example considered here, the linearization condition $\left|H(4 / c)^{n / 2}\right| \ll 1$ translates into $\log _{10}(H) \ll-4.3$. This is consistent with the fact that the linear behavior is observed below -5 . The third part (III), is the region where the polynomial approximation breaks down. Given the approximate form given in Eq. (4.6), this should certainly happen when $\left|H(4 / c)^{n / 2}\right| \approx l_{\max }$. This means $\log _{10}(H) \approx-2.0$ 
in our example. On the figure, one sees that for $\log _{10}(H) \approx-2.4$, the magnetization drops suddenly instead of reaching its asymptotic value at large $H$, namely $\mathcal{M}=1$. Finally, the intermediate region (II) is the one which contains the information we are interested in.

As advertized, we will first take the infinite volume limit of the magnetization at nonzero magnetic field and then extrapolate to zero magnetic field. We need to understand how the second region shown in Fig. 3 changes with $n$. From the above discussion, region II is roughly given by the range of magnetic field

$$
-(n / 2) \log _{10}(4 / c)<\log _{10}(H)<\log _{10}\left(l_{\max }\right)-(n / 2) \log _{10}(4 / c)
$$

In the log scale of Fig. 3, the width of region II is at $\operatorname{most} \log _{10}\left(l_{\max }\right)$ which is approximately 2.3 in our sample calculation. Region II shifts by $-(1 / 2) \log _{10}(4 / c)$, approximately 0.25 in our sample calculation, at each iteration. In addition, the whole graph moves slightly up at each iteration in a way which is better seen using a linear scale as in Fig. 1 .

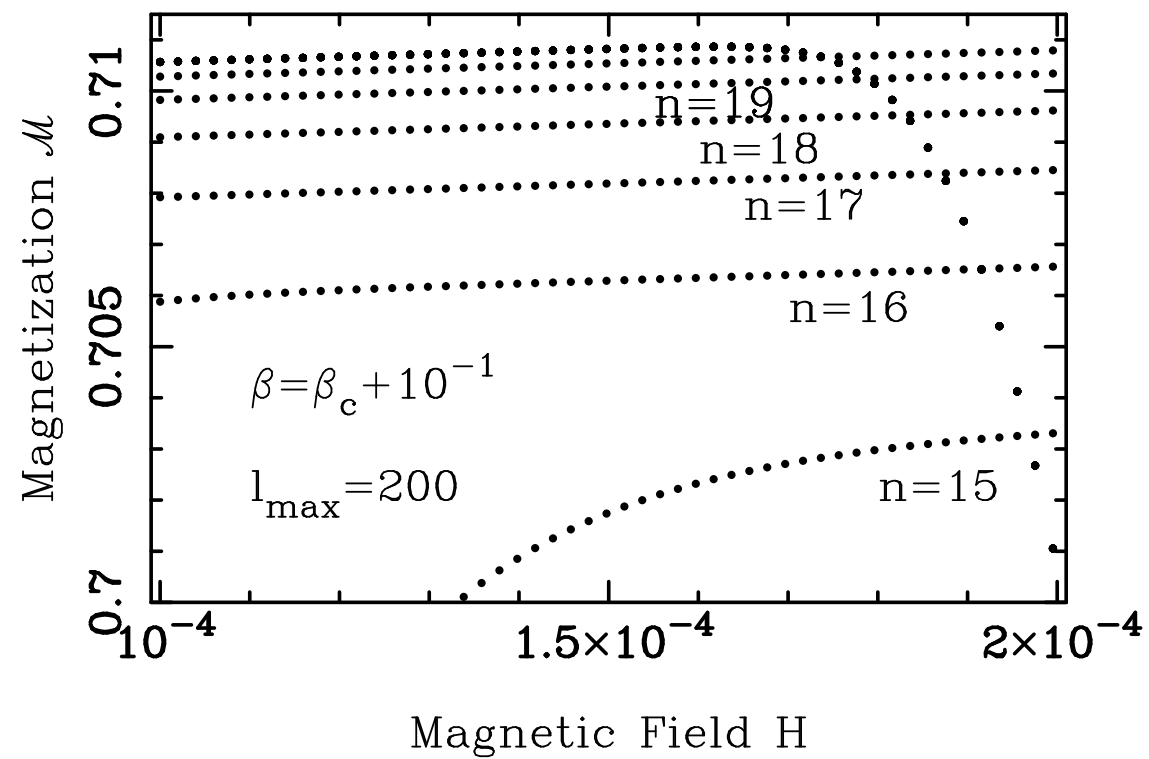

FIG. 4. The magnetization versus the magnetic field for $n=15$ (lower set of point) to 21 (upper set of points on the left of the figure) for $l_{\max }=200$ and $\beta=\beta_{c}+10^{-1}$.

As one can see, the regions II of seven successive iterations do not overlap. Consequently 1.5 is a more realistic estimate than the previously quoted bound 2.3 for the average width of region II. 
The fewer iterations we use to extrapolate to infinite volume, the broader the range of the magnetic field can be. We have compared 5 sets of 4 iterations well on the low-temperature shoulder starting from the set $(17,18,19,20)$ up to the set $(21,22,23,24)$. From our experience in the symmetric phase [6] we have assumed that the finite-size effects could be parametrized as

$$
\mathcal{M}_{n}=\mathcal{M}_{\infty}-A \times B^{n}
$$

This relation implies that

$$
\log _{10}\left(\mathcal{M}_{n+1}-\mathcal{M}_{n}\right)=\tilde{A}+n \times \log _{10}(B)
$$

where $\tilde{A}=\log _{10}(A)+\log _{10}(1-B)$. The value $\tilde{A}$ and $\log _{10}(B)$ can be obtained from linear fits. For four successive iterations, this will give us 3-point fits for the infinite volume limit at fixed value of $H \neq 0$. In all fits performed, we found $B \simeq 0.63$, which is compatible with the $(c / 2)^{n}$ decay of the finite size effects found in the symmetric phase [6]. In terms of the linear dimension $L$ introduced in Eq. (3.17), this corresponds to finite-size effects decaying like $L^{-2}$. If the parametrization of Eq. (5.7) was exact, the value of $\mathcal{M}_{n}+A \times B^{n}$ would be independent of $n$ and equal to $\mathcal{M}_{\infty}$. In practice, variations slightly smaller than $10^{-6}$ are observed. We have thus taken an average over these values in order to estimate $\mathcal{M}_{\infty}$ at fixed $H$. The results for the first set are shown in Fig. 5 for various values of $H$. The linear behavior allows an easy extrapolation to $H=0$. 


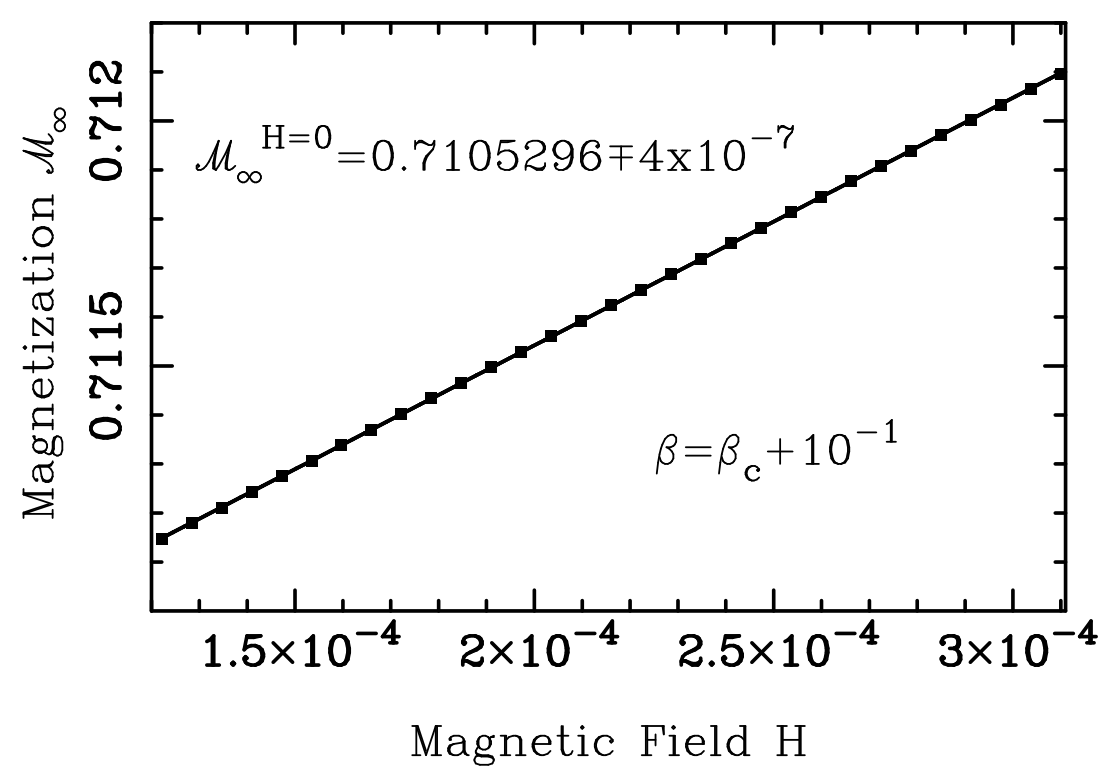

FIG. 5. $\mathcal{M}_{\infty}$ extrapolated from the data for $n=17,18,19$ and 20 , versus the magnetic field for $l_{\max }=200$ and $\beta=\beta_{c}+10^{-1}$.

We have repeated this procedure for the four other set of successive values defined previously and obtained the $H=0$ extrapolations:

\begin{tabular}{lc} 
Set & $\mathcal{M}_{\infty}^{H=0}$ \\
\hline 1 & 0.7105296 \\
2 & 0.7105349 \\
3 & 0.7105376 \\
4 & 0.7105380 \\
5 & 0.7105382
\end{tabular}

Averaging over these five values, we obtain

$$
\mathcal{M}_{\infty}^{H=0}=0.710536 \mp 3 \times 10^{-6}
$$

It may be argued that the values coming from sets involving larger values of $n$ are better estimates because the finite size effects are smaller for those sets. 
We have repeated this type of calculation with sets of 5 successive iterations and a correspondingly narrower range of magnetic field and found results compatible with the estimate given by Eq. (5.9).

\section{The Susceptibility}

We now consider the calculation of the connected susceptibility (two-point function). By using the previous notation, we can express it as

$$
\begin{aligned}
\chi_{n}(H) & =\frac{\left\langle M_{n}\right\rangle_{n, H}^{2}-\left\langle M_{n}^{2}\right\rangle_{n, H}}{2^{n}} \\
& =\frac{\left(b_{1}^{2}-2 \times b_{2}\right)}{2^{n}},
\end{aligned}
$$

where

$$
\frac{R_{n}\left(k+i H(4 / c)^{n / 2}\right)}{R_{n}\left(i H(4 / c)^{n / 2}\right)}=\sum_{q=0}^{\infty} b_{q} k^{q} .
$$

The dependence on $H$ of the $b_{n}$ is implicit.

In order to extrapolate the susceptibility to infinite $n$, one has to determine the range of the magnetic field for which the scaling $\left\langle M_{n}\right\rangle_{n, H}^{2}-\left\langle M_{n, H}^{2}\right\rangle_{n} \propto 2^{n}$ holds. When this is the case, the ratio $\chi_{n+1} / \chi_{n} \simeq 1$. The range of values of the magnetic field for which this scaling is observed is analogous to "region II" introduced in the previous subsection, and we will use the same terminology here. The ratios of the susceptibility at successive $n$ are shown for various values of $H$ in Fig. 6 . 


$$
\beta-\beta_{c}=10^{-1}
$$

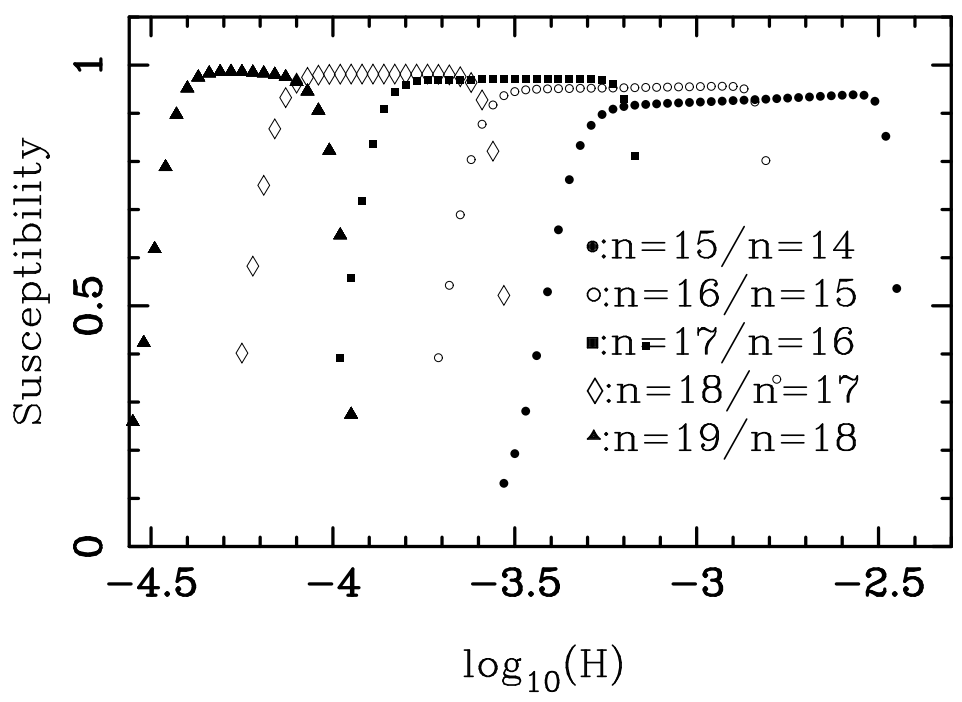

FIG. 6. The successive ratios of the susceptibility for $\beta=\beta_{c}+10^{-1}$.

One observes that the range where the desired scaling is observed shrinks when $n$ increases. For each successive iteration, the ratio of susceptibility has a "upside-down U"shape. The values of $H$ for which the ratio starts dropping on the left are equally spaced and can be determined by linearization as before. On the other side of the upside-down $\mathrm{U}$, dropping values of the ratio signal the breakdown of the polynomial truncation. This occurs at smaller values of $H$ than for the magnetization, making the region II smaller. A theoretical estimate of the lower value of $H$ for which this happens requires a more refined parametrization than the one given in Eq. (4.6). In order to get a controllable extrapolation, we need at least 4 successive values of $\chi_{n}$ (to get at least 3-point fits for the logarithm of the differences). This is unfortunately impossible: the region II of three successive upside-down U have no overlap as one can see from Fig. 6. Similar results are obtained by plotting the susceptibility versus the magnetic field as shown in Fig. 7. 


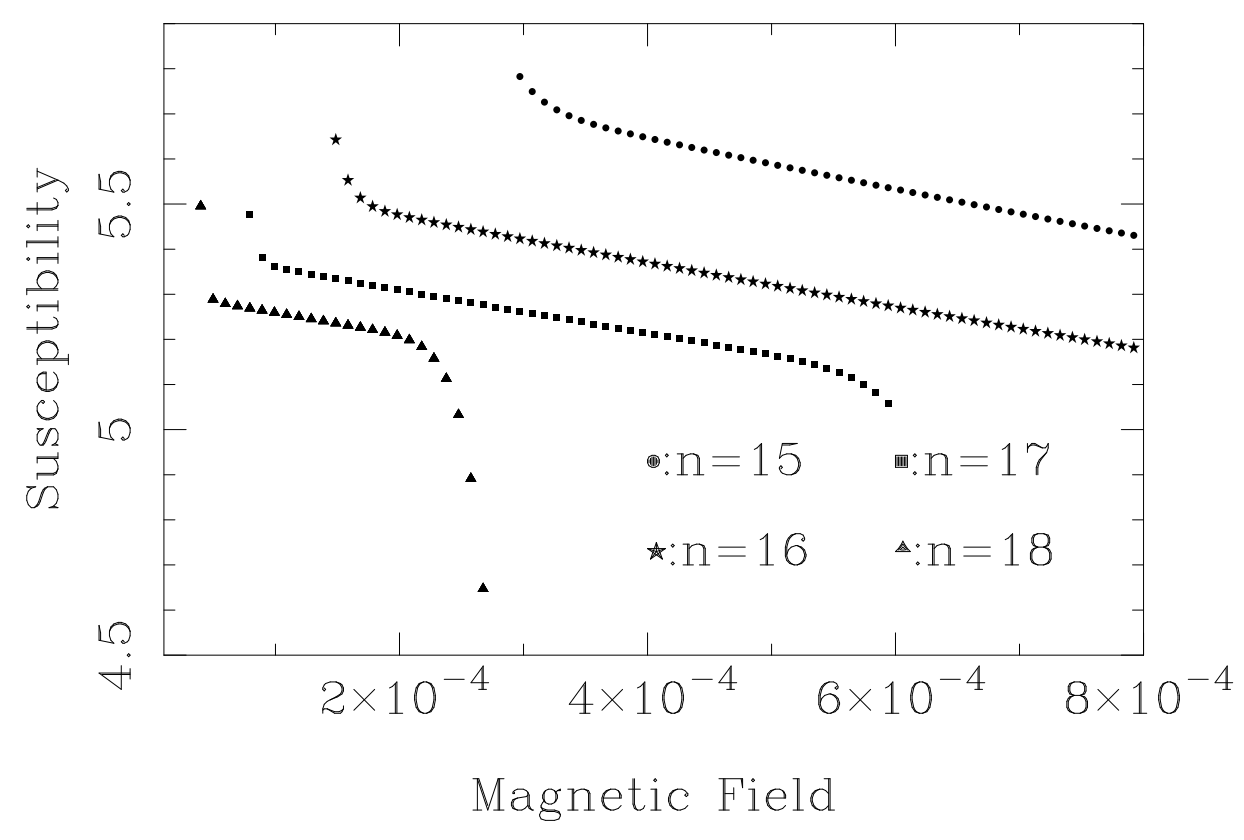

FIG. 7. The susceptibility $\chi_{n}$ versus the magnetic field $H$ at different $n$ for $\beta=\beta_{c}+10^{-1}$.

One sees that the regions II (where the susceptibility can approximately be fitted by a line which extrapolates to a non-zero value at zero $H$ ) do not overlap for 4 consecutive iterations.

\section{An Alternate Method}

In the previous discussion, we have observed a linear behavior for the region II of the magnetization and the susceptibility. This linear behavior can be used to obtain extrapolations to non-zero values of these quantities at zero magnetic field. These values have no physical interpretation. We denote them by $\mathcal{M}_{n}^{H \rightarrow 0}$, the arrow indicating that the quantity is a mathematical extrapolation and not "the spontaneous magnetization at finite volume". They reach an asymptotic value at an exponential suppressed rate when $n$ increases, just as in Eq. (5.7). This is illustrated in Fig. 8. 


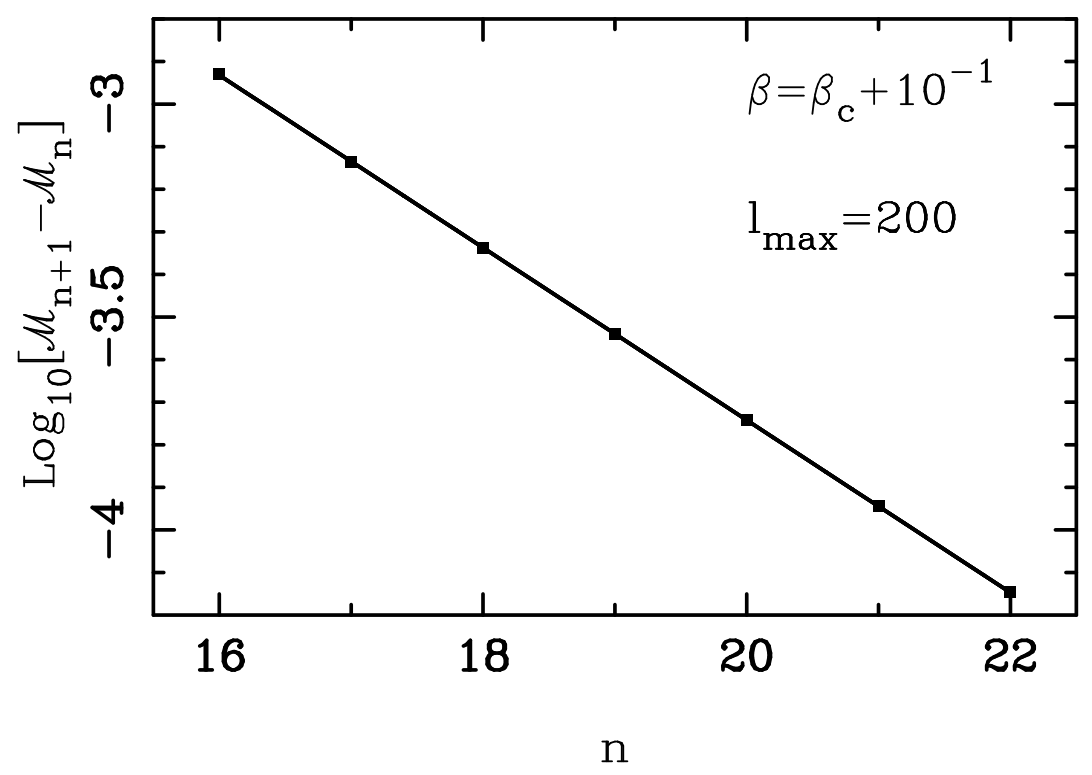

FIG. 8. $\log _{10}\left(\mathcal{M}_{n+1}^{H \rightarrow 0}-\mathcal{M}_{n}^{H \rightarrow 0}\right)$ versus $n$ for $l_{\text {max }}=200$ and $\beta=\beta_{c}+10^{-1}$.

Using a linear fit to fix the unknown parameters $A$ and $B$ in Eq. 5.7, and averaging the $\mathcal{M}_{n}^{H \rightarrow 0}+A B^{n}$ over $n$, we obtain

$$
\mathcal{M}_{\infty}^{H=0}=0.710537 \mp 2 \times 10^{-6}
$$

which is consistent with the result obtained with the standard method.

Roughly speaking, the lines of region II move parallel to each other when $n$ increases and it is approximately equivalent to first extrapolate to zero $H$, using the linear behavior in region II, and then to infinite $n$ rather than the contrary. If the two limits coincide, the second method has a definite practical advantage: all we need is a small part of region II for each $n$, no matter if it overlaps or not with the region II for other $n$. So in general, it allows to use more iterations to get better quality extrapolations. The fact that the values of the magnetization obtained with the two methods coincide with 5 significant digits is a strong indication that the two procedure are equivalent. For the susceptibility and higher point functions, we do not have an independent check, since the alternate method is the only one available. However, we were able to make consistency checks such as the fact that the slope of the straight line used for the zero magnetic field extrapolation of the $q$-point function 
coincides with the $q+1$-point function.

We can repeat the same steps for the 3-point function. The 3-point function is given by

$$
\begin{aligned}
G_{3}^{c} & =\frac{M_{3}-3 M_{1} M_{2}+2 M_{1}^{3}}{2^{n}} \\
& =\frac{6 b_{3}-6 b_{1} b_{2}-2 b_{1}^{3}}{2^{n}},
\end{aligned}
$$

where the dependence on $H$ is implicit. As shown in Ref. [17], $G_{3}^{c}<0$ for $H \leq 0$. Due to the additional subtraction, the range where the proper low-temperature scaling is observed is smaller than for the suceptibility. It is not possible to repeat the same steps for the 4-point function which is given by,

$$
G_{4}^{c}=M_{4}-3 M_{2}^{2}-4 M_{1} M_{3}+12 M_{1}^{2}-6 M_{1}^{4},
$$

and involves one more subtraction.

\section{ESTIMATION OF THE EXPONENTS}

We have used the method described in the previous section to calculate the value of the connected $q$-point functions at value of $\beta$ approaching $\beta_{c}$ from above with equal spacings on a logarithmic scale. For reference, the numerical values are given in the table below.

\begin{tabular}{cccc}
\hline$-\log _{10}\left(\beta-\beta_{c}\right)$ & $G_{1}^{c}(0)$ & $G_{2}^{c}(0)$ & $G_{3}^{c}(0)$ \\
\hline 1 & 0.710537 & 5.1449 & -452 \\
2 & 0.372929 & 147.75 & $-4.83 \times 10^{6}$ \\
3 & 0.181173 & 3270.0 & $-4.42 \times 10^{8}$ \\
4 & $8.64639 \times 10^{-2}$ & 67534 & $-3.82 \times 10^{11}$ \\
5 & $4.10479 \times 10^{-2}$ & $1.3628 \times 10^{6}$ & $-3.23 \times 10^{14}$ \\
6 & $1.94518 \times 10^{-2}$ & $2.7276 \times 10^{7}$ & $-2.72 \times 10^{17}$ \\
7 & $9.21183 \times 10^{-3}$ & $5.4411 \times 10^{8}$ & $-2.28 \times 10^{20}$ \\
8 & $4.36138 \times 10^{-3}$ & $1.0842 \times 10^{10}$ & $-1.91 \times 10^{23}$
\end{tabular}




\begin{tabular}{cccc}
9 & $2.06473 \times 10^{-3}$ & $2.1596 \times 10^{11}$ & $-1.60 \times 10^{26}$ \\
10 & $9.77434 \times 10^{-4}$ & $4.3010 \times 10^{12}$ & $-1.34 \times 10^{29}$ \\
11 & $4.62716 \times 10^{-4}$ & $8.5641 \times 10^{13}$ & $-1.11 \times 10^{32}$ \\
12 & $2.19084 \times 10^{-4}$ & $1.7042 \times 10^{15}$ & $-9.40 \times 10^{34}$ \\
\hline
\end{tabular}

The estimated errors on the values quoted above are of order 1 in the last digit for the first lines of the table and slowly increase when one moves down the table. For the last lines, the effects of the round-off errors become sizable. Otherwise, the errors are mainly due to the extrapolation procedure. We have checked that the numerical values of the quantities at finite $H$ had reaches their asymptotic values (well within the accuracy of the final result) as a function of $l_{\max }$.

The results are displayed in Fig. 9 in a log-log plot.

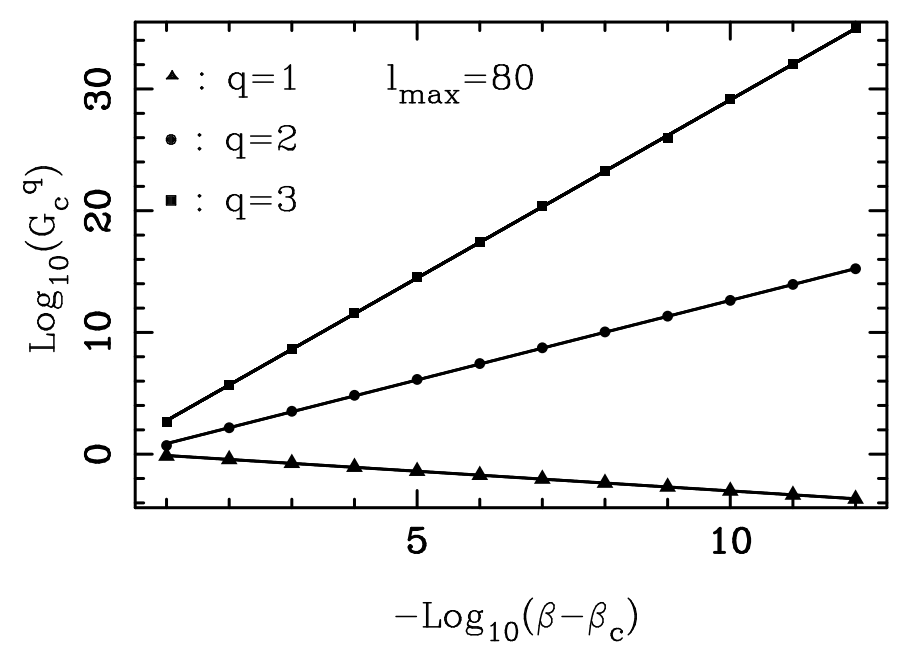

FIG. 9. $\log _{10}\left(G_{c}^{q}(0)\right)$ versus $\log _{10}\left(\beta-\beta_{c}\right)$ for $q=1,2$ and 3 .

The departure from the linear behavior is not visible on this figure. In the symmetric phase, we know [7] that the relative strength of the subleading corrections is approximately $-0.57\left(\beta_{c}-\beta\right)^{0.43}$. It is likely that a similar behavior should be present in the low-temperature phase. Consequently, taking into account the data on the left part of Fig. 9 will distort the value of the exponents. On the other hand getting too close to criticality generates large 
numerical errors. Using a linear fit of the data starting with the 5-th point and ending with the 10-th point, we obtain the value of the exponents

$$
\begin{aligned}
& \gamma_{1}=-0.3247 \\
& \gamma_{2}=1.2997 \\
& \gamma_{3}=2.9237 .
\end{aligned}
$$

This can be compared with the predictions from scaling and hyperscaling given by Eq. (1.1) and amount numerically to:

$$
\begin{aligned}
& \gamma_{1}=-0.324785 \\
& \gamma_{2}=1.2991407 \\
& \gamma_{3}=2.923066
\end{aligned}
$$

Better estimates can be obtained by using the method developed in Ref. [7], where it was found that the combined effects of the two types of errors are minimized for $10^{-10}<\left|\beta_{c}-\beta\right|<$ $10^{-9}$. This allowed estimates of $\gamma$ (in the symmetric phase) with errors of order $3 \times 10^{-5}$ (compared to more accurate estimates). Using 10 values between $10^{-9}$ and $10^{-10}$ with equal spacing on a logarithmic scale, we obtain here

$$
\begin{aligned}
& \gamma_{1}=-0.324775 \pm 2 \times 10^{-5} \\
& \gamma_{2}=1.29918 \pm 10^{-4} \\
& \gamma_{3}=2.928 \pm 10^{-2}
\end{aligned}
$$

The errors due to the subleading corrections and the round-off errors are approximately of the same order in this region of temperature [7]. The errors due to the subleading corrections are larger for larger values of $\left|\beta_{c}-\beta\right|$ while the numerical errors are larger for smaller values of $\left|\beta_{c}-\beta\right|$. We have estimated the errors due to the subleading corrections by performing the same calculation between $10^{-8}$ and $10^{-9}$. The errors bars quoted above reflect the differences with the exponents obtained in this second region. 


\section{CONCLUSIONS}

One sees clearly that our best estimates of the critical exponents (Eq. (6.3)) are fully compatible with the predictions of hyperscaling (Eq. (6.2)). The differences between the predicted and calculated values are $10^{-5}$ for $\gamma_{1}, 4 \times 10^{-5}$ for $\gamma_{2}$ and $5 \times 10^{-3}$ for $\gamma_{3}$. They fall well within the estimated errors. Since hyperscaling is a reasonable expectation, this also shows that the non-standard extrapolation method that we have used is reliable. As far as $\gamma_{1}$ and $\gamma_{2}$ are concerned, the errors bars are smaller than what can usually be reached using a series analysis or Monte Carlo simulation. Our result for $\gamma_{1}$ is also compatible with the result 0.325 obtained in Ref. [16] for the hierarchical model (for $\sigma / d=2 / 3$ with their notations) using the integral formula.

One could in principle improve the accuracy of these calculations by increasing the size of the polynomial truncation. However, the efficiency of this procedure (errors decreasing like the inverse of the CPU time) is not compatible with our long term objectives (errors decreasing exponentially). The main obstruction to keep using the polynomial truncation is that the generating function $R_{n}(k)$ starts oscillating rapidly in the low-temperature phase making the approximation of the exponential of the Laplacian by a sum inaccurate. It is thus important to obtain an approximate parametrization of $R_{n}(k)$ in terms of eigenfunctions of the Laplacian. A step in this direction is made by the parametrization of Eq. (4.6). This approximate analytical form needs to be improved in order to include the connected 2-point and higher point functions in terms of an exponential function. This possibility is presently under investigation.

This research was supported in part by the Department of Energy under Contract No. FG02-91ER40664.

[1] M. Caselle, M. Hasenbush and P. Provero, hep-lat/9907018, to appear in the Proceedings of Lattice 99. 
[2] P. Cea, M. Consoli and L. Cosmai, hep-lat/9909055, to appear in the Proceedings of Lattice 99.

[3] J. Engels and T. Mendes, hep-lat/9909013, to appear in the Proceedings of Lattice 99.

[4] K. Wilson, Phys. Rev. B. 4, 3185 (1971) ; Phys. Rev. D. 3, 1818 (1971); K. Wilson and J. Kogut Phys. Rep. 12, 75 (1974); K.Wilson, Phys. Rev. D 6, 419 (1972).

[5] F. Dyson, Comm. Math. Phys. 12, 91 (1969) ; G. Baker, Phys. Rev. B5, 2622 (1972).

[6] J. J. Godina, Y. Meurice, B. Oktay and S. Niermann, Phys. Rev. D 57, 6326 (1998).

[7] J. J. Godina, Y. Meurice, B. Oktay, Phys. Rev. D 57, R6581 (1998); Rev. D 59, 096002 (1999).

[8] H. Koch and P. Wittwer, Math. Phys. Electr. Jour. (http://mpej.unige.ch/), 1, Paper 6 (1995).

[9] M. Fisher, in Critical Phenomena, Lecture Notes in Physics 186, Edited by F. Hahne (SpringerVerlag, Berlin 1983).

[10] P. Bleher and Y. Sinai, Comm. Math. Phys. 45, 247 (1975) ; P. Collet and J. P. Eckmann, Comm. Math. Phys. 55, 67 (1977); H. Koch and P. Wittwer, Comm. Math. Phys. 106495 (1986), Ibid. 138 (1991) 537 , Ibid. 164 (1994) 627.

[11] H. E. Stanley, Introduction to Phase Transitions and Critical Phenomena (Oxford University Press, New York, 1971).

[12] M. Fisher and J. Chen, J. Phys. (Paris) 46, 1645 (1985).

[13] Y. Meurice and S. Niermann, Phys. Rev. E 60, 2612 (1999)

[14] Y. Meurice, G. Ordaz and V. G. J. Rodgers, Phys. Rev. Lett. 75, 4555 (1995) . Y. Meurice, S. Niermann, and G. Ordaz, J. Stat. Phys. 87, 363 (1997).

[15] K. Rajagopal and F. Wilczek, Nucl. Phys. B 399, 395 (1993).

[16] G. Baker and G. Golner, Phys. Rev. B 16, 2801 (1977). 
[17] J. Glimm and A. Jaffe, Quantum Physics (Springer-Verlag, New York, 1987). 


\section{SPONTANEOUS MAGNETIZATION}

In this section, we will explain how to calculate the magnetization. We will do this in two different ways. One of them is to reach to infinite volume limit at different magnetic fields and then extrapolate to zero magnetic field and find the spontaneous magnetization. The second method is to obtain the spontaneous magnetization first and then take the infinite volume limit.

The major difficulty which force us to go to the second method is the limitations of the polynomial truncation. In the low temperature phase, we have a very limited shoulder. One way of increasing the number of the points on this shoulder is to increase $l_{\max }$ but this will not be possible in the double precision Fortran if we want to go beyond $l_{\max }=80$. For this reason, we will use Mathematica where we can set $l_{\max }=200$. This will be very important when we want to take the infinite volume limit first. This shoulder is shown in the Fig. ?? below for $l_{\max }=80$ and $l_{\max }=200$.

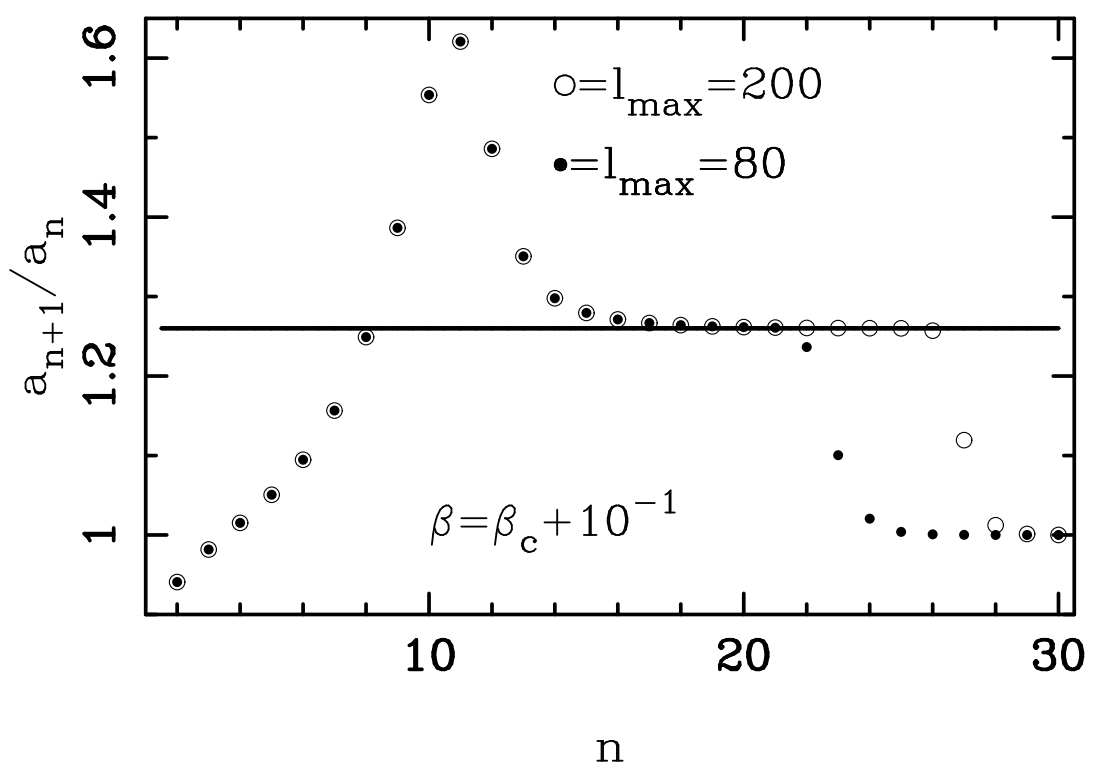

FIG. 1. Shoulder at $\beta=\beta_{c}+10^{-1}$. $l_{\max =200}$ (empty circles) and $l_{\max }=80$. (filled circles)

Before we go into the details,we need to discuss the change of the magnetization with 
respect to magnetic field. We will obtain the infinite volume limit at different magnetic field values. The shape of the magnetization is shown in the Fig.??.

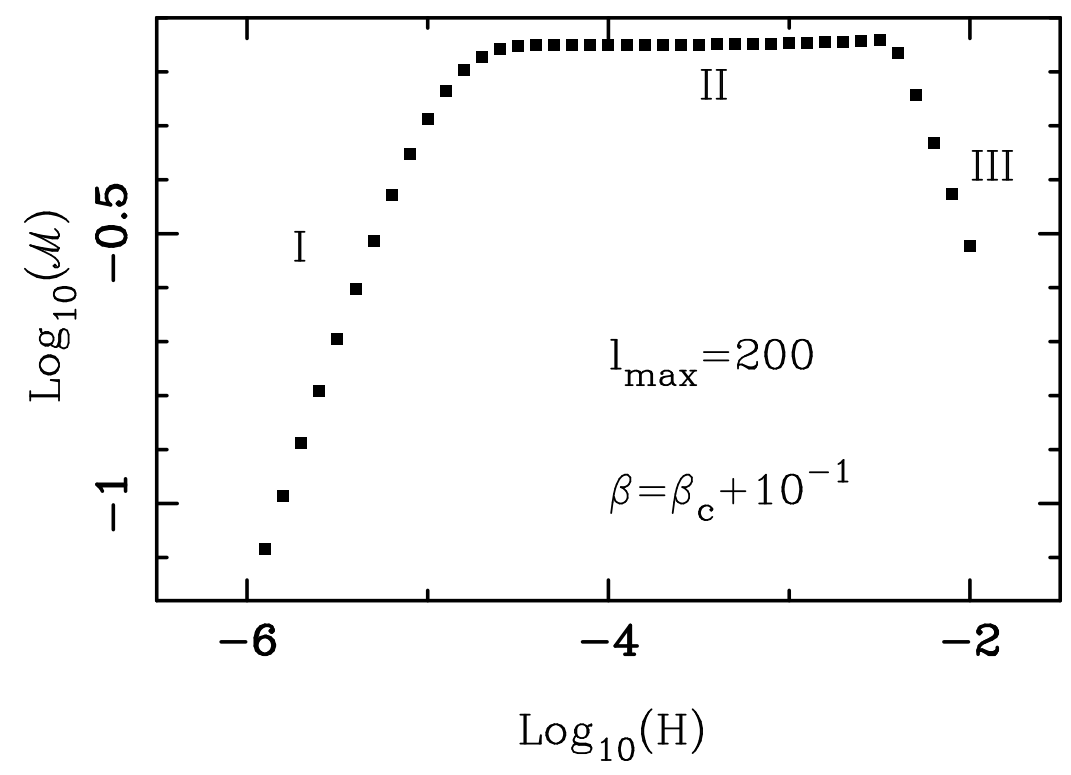

FIG. 2. $\log _{10}(\mathcal{M})$ versus $\log _{10}(H)$ at $n=16$ for $\beta=\beta_{c}+10^{-1}$.

As one can see, we have three different region. The first one goes linearly to zero where the system is not magnetized and we have weak field. The second part is which we are going to use to reach infinite volume limit and the third one is the part where the polynomial truncation stops being adequate.

II. $\mathcal{L}_{M A X}=200$

\section{A. From infinite volume to spontaneous magnetization}

In this subsection, we will first obtain the infinite volume limit of the magnetization and then make extrapolation to the zero magnetic field to obtain the spontaneous magnetization. The region we use is the second region shown in Fig.?? above. Since we are going to take the limit first, we should have this region for all shoulder points. Unfortunately, it will not be the case. This region will shift and give us a different part for each shoulder point. In 
this case, It will not be possible to reach to the infinite volume limit. However, there will be an overlapping area for 4 successive point and the fifth point will be out of this range. This is shown in Fig.?? below.

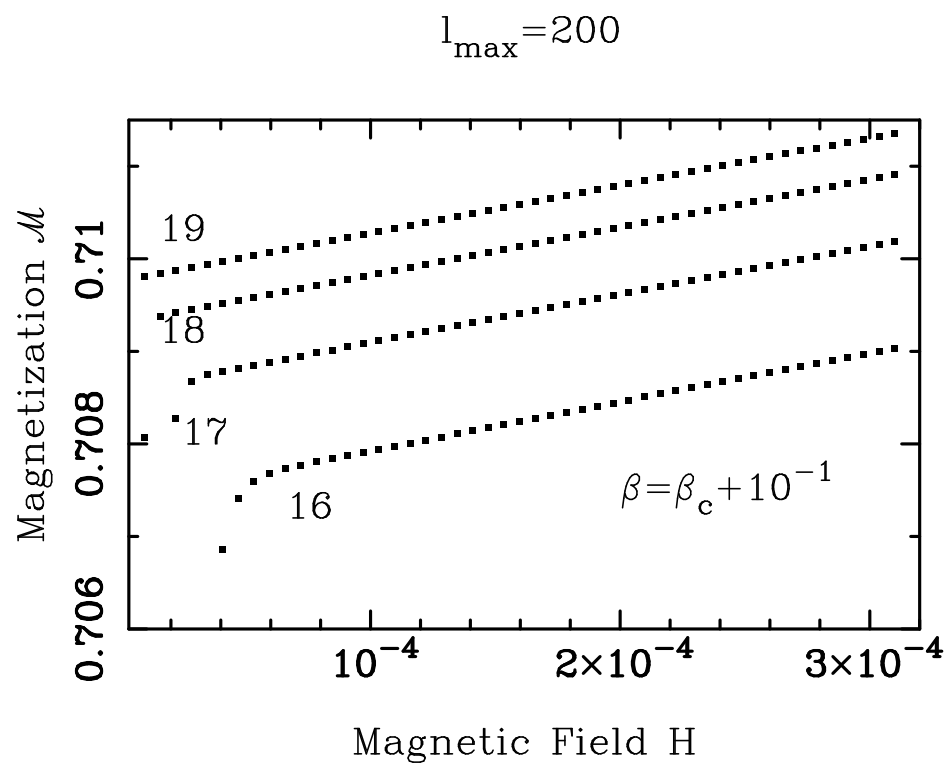

FIG. 3. Magnetization vesus magnetic field for $n=16,17,18,19$ for $l_{\max }=200$ and $\beta=\beta_{c}+10^{-1}$.

For $l_{\max }=200$, our shoulder points goes from 16 to 25 and since we can only have 4 points in a linear region to take the limit we make sets of four points starting from 16,17, 18, 19 the first set, $17,18,19,20$ the second set and so on until $22,23,24,25$. On the other hand the points 24 and 25 are at the end of the shoulder and for these points we will not be able to see the second region. Then, we will work with the first 5 sets. These four point will give us 3 -point fits for the infinite volume limit at $H \neq 0$.

\section{B. Accuracy of the 3-point fits}

We would like to know the accuracy of out three point fits. We will assume the form of

$$
\mathcal{M}_{n}=\mathcal{M}_{\infty}-A \times B^{n}
$$


and from this relation,

$$
\log _{10}\left(\mathcal{M}_{n+1}-\mathcal{M}_{n}\right)=\tilde{A}+n \times \log _{10}(B)
$$

where $\tilde{A}=\log _{10}(A)+\log _{10}(1-B)$. We listed the errors on the three-point fits for the first set at different magnetic fields below.

\begin{tabular}{|c|c|c|c|c|c|}
\hline Magnetic Field & $\tilde{A}$ & $\delta \tilde{A}$ & $\log _{10}(B)$ & $\delta \log _{10}(B)$ & $\sigma_{f i t}$ \\
\hline 0.0001221271676601683 & 0.356154 & 0.0110876 & -0.205475 & 0.00065146 & 0.000921303 \\
0.0001346497876601683 & 0.355549 & 0.0110646 & -0.205466 & 0.00065011 & 0.000919394 \\
0.0001471724076601683 & 0.354946 & 0.0110421 & -0.205457 & 0.000648786 & 0.000917522 \\
0.0001596950276601682 & 0.354345 & 0.0110197 & -0.205448 & 0.000647472 & 0.000915663 \\
0.0001722176476601682 & 0.353746 & 0.0109975 & -0.205439 & 0.000646165 & 0.000913815 \\
0.0001847402676601683 & 0.353148 & 0.0109754 & -0.20543 & 0.000644866 & 0.000911978 \\
0.0001972628876601682 & 0.352552 & 0.0109534 & -0.205421 & 0.000643574 & 0.000910151 \\
0.0002097855076601684 & 0.351958 & 0.0109315 & -0.205413 & 0.000642289 & 0.000908334 \\
0.0002223081276601682 & 0.351365 & 0.0109098 & -0.205404 & 0.000641012 & 0.000906528 \\
0.0002348307476601682 & 0.350773 & 0.0108881 & -0.205395 & 0.000639741 & 0.000904731 \\
0.0002473533676601682 & 0.350184 & 0.0108666 & -0.205386 & 0.000638478 & 0.000902945 \\
0.0002598759876601683 & 0.349595 & 0.0108453 & -0.205378 & 0.000637222 & 0.000901168 \\
0.0002723986076601683 & 0.349009 & 0.010824 & -0.205369 & 0.000635972 & 0.0008994 \\
0.0002849212276601683 & 0.348424 & 0.0108024 & -0.205361 & 0.000634705 & 0.000897609 \\
0.0002974438476601681 & 0.347854 & 0.0107735 & -0.205353 & 0.000633007 & 0.000895207 \\
0.0003099664676601682 & 0.347461 & 0.0106387 & -0.205356 & 0.000625088 & 0.000884008 \\
\hline
\end{tabular}

From these values, we then calculate the $\mathcal{M}_{\infty}$ at each different magnetic field value. Finally, we fit all these $\mathcal{M}_{\infty}$ values to obtain the spontaneous magnetization in the infinite volume limit. This is show in the Fig.??. below. 


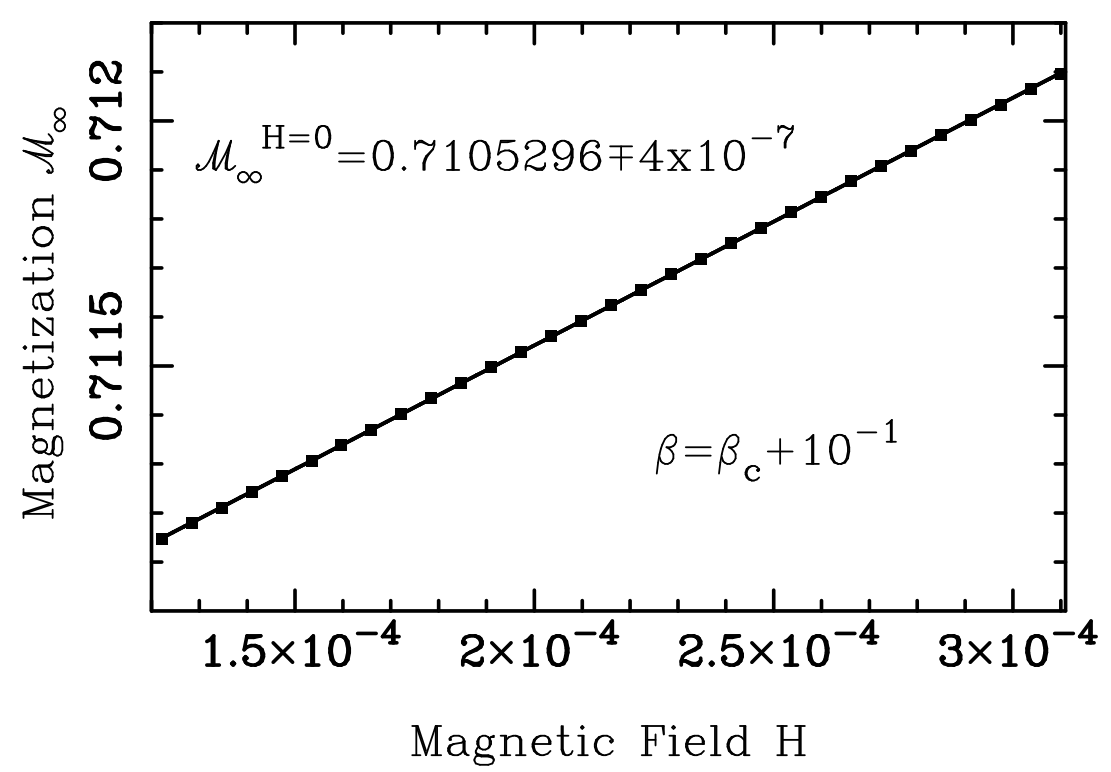

FIG. 4. $\mathcal{M}_{\infty}$ versus magnetic field for $n=16,17,18,19$ for $l_{\max }=200$ and $\beta=\beta_{c}+10^{-1}$ and the extrapolation to the zero magnetic field.

\begin{tabular}{lll} 
Set & $\mathcal{M}_{\infty}^{H=0}$ & $\delta \mathcal{M}_{\infty}^{H=0}$ \\
\hline 1 & 0.7105296 & $4.6 \mathrm{E}-7$ \\
2 & 0.7105349 & $8.2 \mathrm{E}-7$ \\
3 & 0.7105376 & $8.5 \mathrm{E}-7$ \\
4 & 0.7105380 & $1.7 \mathrm{E}-7$ \\
5 & 0.7105382 & $4.5 \mathrm{E}-7$
\end{tabular}

From these data points we conclude that

$$
\mathcal{M}_{\infty}^{H=0}=0.710536 \mp 3.25 \times 10^{-6}
$$




\section{From extrapolation to the infinite volume limit}

In this subsection, we would like to extrapolate to the spontaneous magnetization and then take the infinite volume limit and then compare our result with the one we obtained by using the first method above. This comparison will be handy when we use double precision arithmetic with $l_{\max }=80$. This procedure is quite simple. We use all the possible shoulder points we have and then from the linear region we make an extrapolation to the zero magentic field. The results for each $n$ are given in the table below. For each fit

\begin{tabular}{lcc}
$n$ & $\mathcal{M}_{H=0}$ & $\delta \mathcal{M}_{H=0}$ \\
\hline 16 & 0.7073963579884217 & $4.91 \mathrm{E}-7$ \\
17 & 0.708571938893955 & $1.46 \mathrm{E}-7$ \\
18 & 0.7093049288248253 & $4.71 \mathrm{E}-8$ \\
19 & 0.7097633112129702 & $4.38 \mathrm{E}-9$ \\
20 & 0.7100511622588243 & $1.26 \mathrm{E}-9$ \\
21 & 0.7102319520698038 & $1.24 \mathrm{E}-9$ \\
22 & 0.7103455954378881 & $1.08 \mathrm{E}-9$ \\
23 & 0.7104170824449012 & $1.08 \mathrm{E}-9$
\end{tabular}

Now, we will use these data above to estimate the infinite volume limit. We will again use

the form of

$$
\mathcal{M}_{n}=\mathcal{M}_{\infty}-A \times B^{n}
$$

This will lead us a 7-point fit which is shown in the Fig.?? below. 


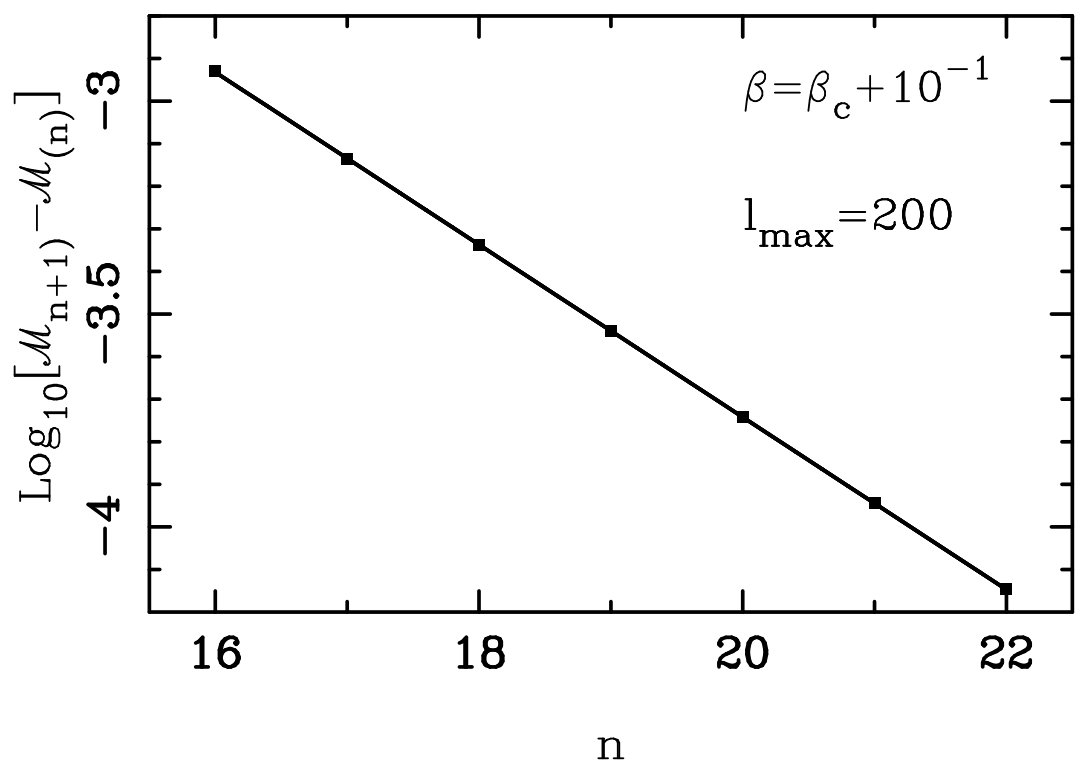

FIG. 5. $\log _{10}\left(\mathcal{M}_{n+1}^{H=0}-\mathcal{M}_{n}^{H=0}\right)$ versus $n$ for $l_{\text {max }}=200$ and $\beta=\beta_{c}+10^{-1}$ and the extrapolation to the infinite volume limit.

We found that $\sigma_{f i t}=1.5 \times 10^{-3}$. From this linear fit of the form $\log _{10}\left(\mathcal{M}_{n+1}^{H=0}-\mathcal{M}_{n}^{H=0}\right)=$ $\tilde{A}+n \log _{10}(B)$ we found that $\log _{10} B=-0.2025442151072931 \mp 0.000295107922264223$ which gives $B=0.6272718307858014 \simeq 0.63$ and $\tilde{A}=\log _{10} A+\log _{10}(1-B)=$ $0.3087245768455599 \mp 0.00563802893830401$. From the last relation, we find $A=$ 5.461756833437532. Then, all we need to do is to put these values back into the equation above and calculate the spontaneous magnetization $\mathcal{M}_{\infty}^{H=0}$. We did this for each $n$ value we have the results are listed in the table below.

\begin{tabular}{lc}
$n$ & $\mathcal{M}_{\infty}^{H=0}$ \\
\hline 16 & 0.7105341555342905 \\
17 & 0.7105401909051874 \\
18 & 0.7105395578673588 \\
19 & 0.7105377592328215 \\
20 & 0.7105369516860849 \\
21 & 0.7105366740932179
\end{tabular}


By using these data point, we conclude (with the simple average and standard deviation ralations) that

$$
\mathcal{M}_{\infty}^{H=0}=0.710537 \mp 1.74 \times 10^{-6}
$$

\section{D. conclusion for $l_{\max }=200$}

By comparing the two results obatined by two different ways for $l_{\max }=200$ and $\beta=$ $\beta_{c}+10^{-1}$, we immedeately see that there is 5 significant digits accuracy between them. Thus, our final result for the spontaneous magnetization at the infinite volume limit is

$$
\mathcal{M}_{\infty}^{H=0}=0.710536 \mp 1 \times 10^{-6}
$$




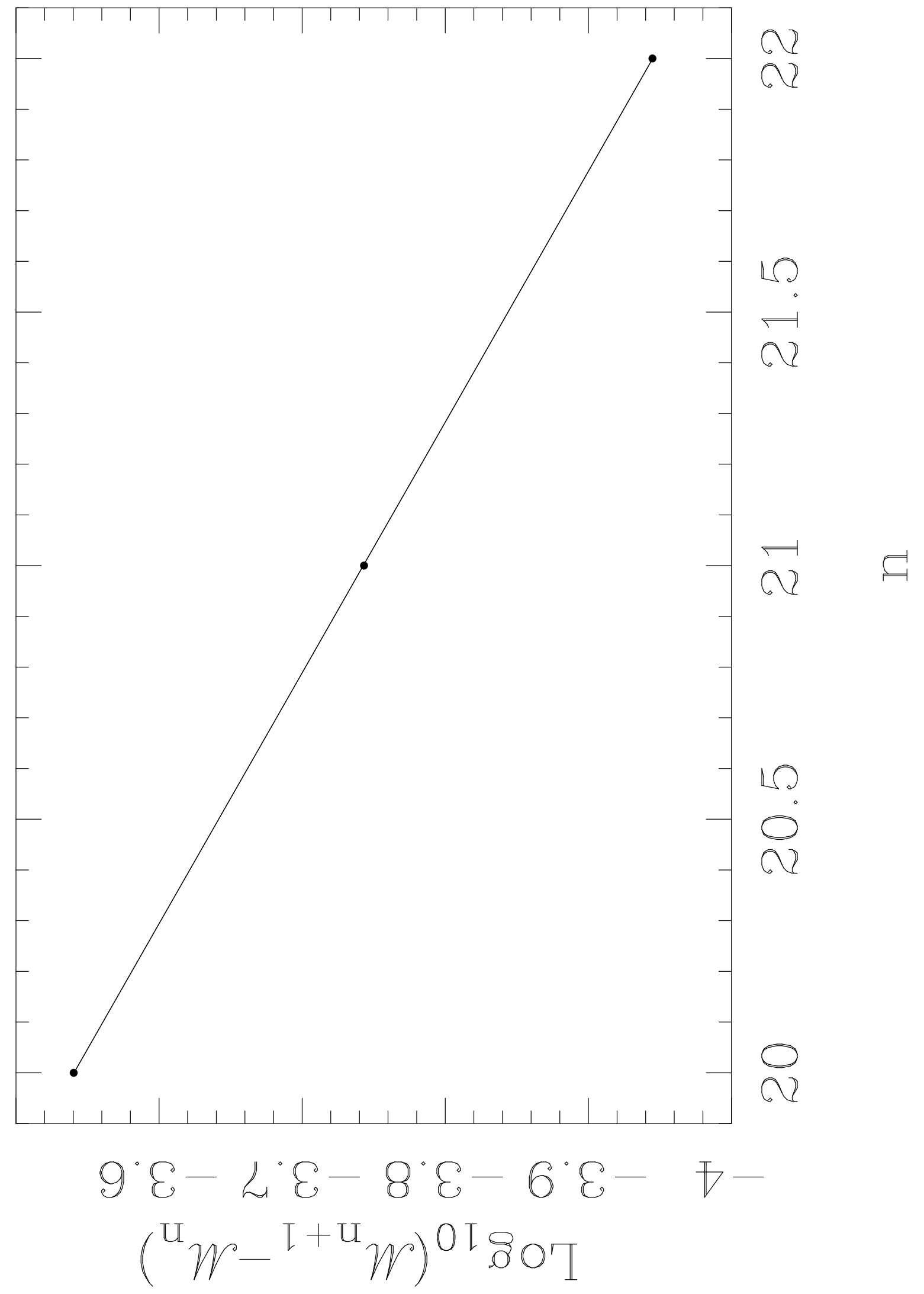




\begin{tabular}{cccc}
\hline$-\log _{10}\left(\beta-\beta_{c}\right)$ & $G_{1}^{c}(0)$ & $G_{2}^{c}(0)$ & $G_{3}^{c}$ \\
\hline 1 & 0.710537 & 5.1449464 & -451.801295 \\
2 & 0.372929 & 147.744937 & -483052.394150 \\
3 & 0.181173 & 3270.020749 & $-4.419043 E 8$ \\
4 & 0.0864639 & 67534.150592 & $-3.817470 \times 10^{11}$ \\
5 & 0.0410479 & $1.362846 \times 10^{6}$ & $-3.231856 \times 10^{14}$ \\
6 & 0.0194518 & $2.727356 \times 10^{7}$ & $-2.720537 \times 10^{17}$ \\
7 & 0.00921183 & $5.441076 \times 10^{8}$ & $-2.280423 \times 10^{20}$ \\
8 & 0.00436138 & $1.084248 \times 10^{10}$ & $-1.914112 \times 10^{23}$ \\
9 & 0.00206473 & $2.159631 \times 10^{11}$ & $-1.597785 \times 10^{26}$ \\
10 & 0.000977434 & $4.301016 \times 10^{12}$ & $-1.343532 \times 10^{29}$ \\
11 & 0.000462716 & $8.564077 \times 10^{13}$ & $-1.113270 \times 10^{32}$ \\
12 & 0.000219084 & $1.704184 \times 10^{15}$ & $-9.407884 \times 10^{34}$ \\
\hline & & &
\end{tabular}




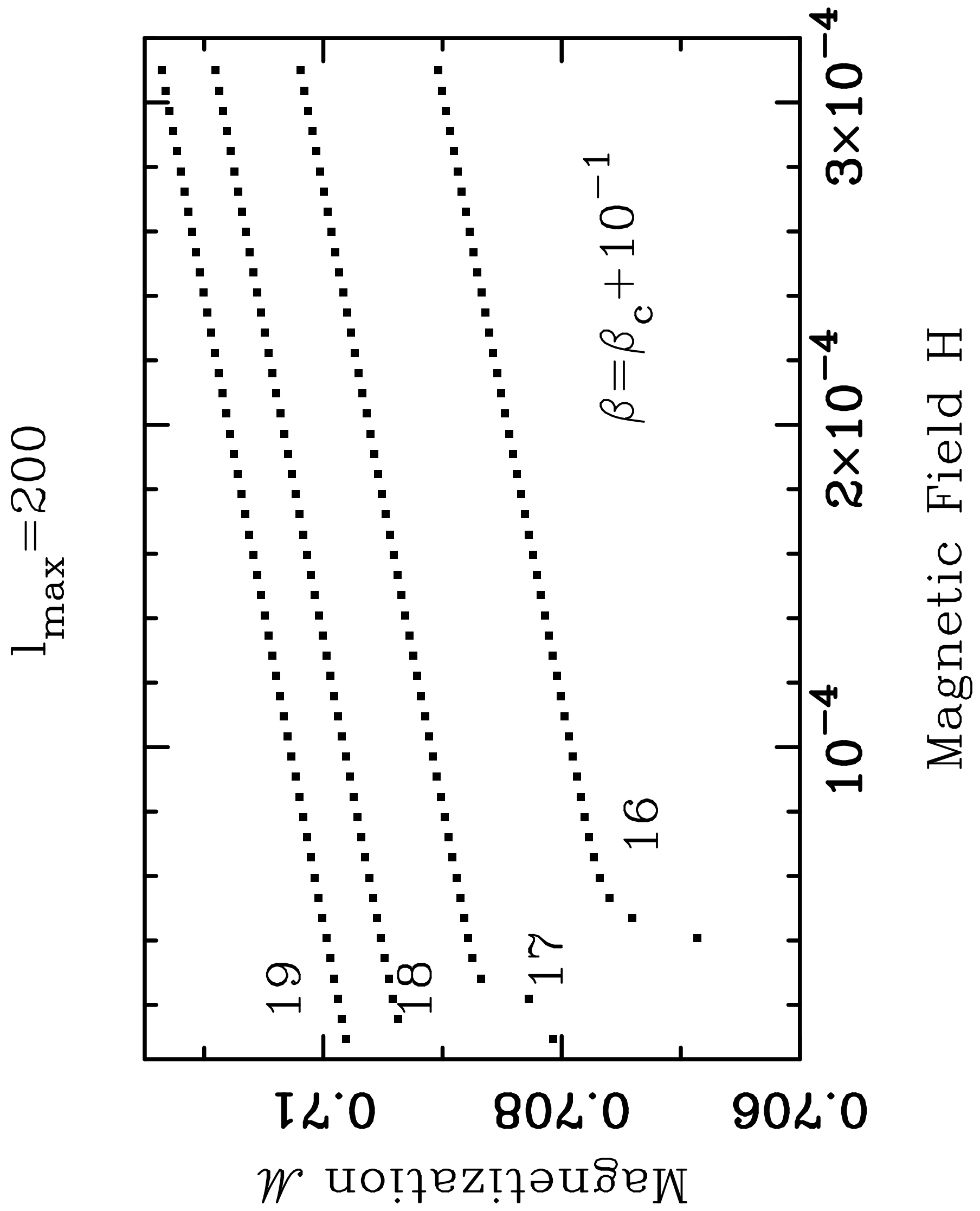




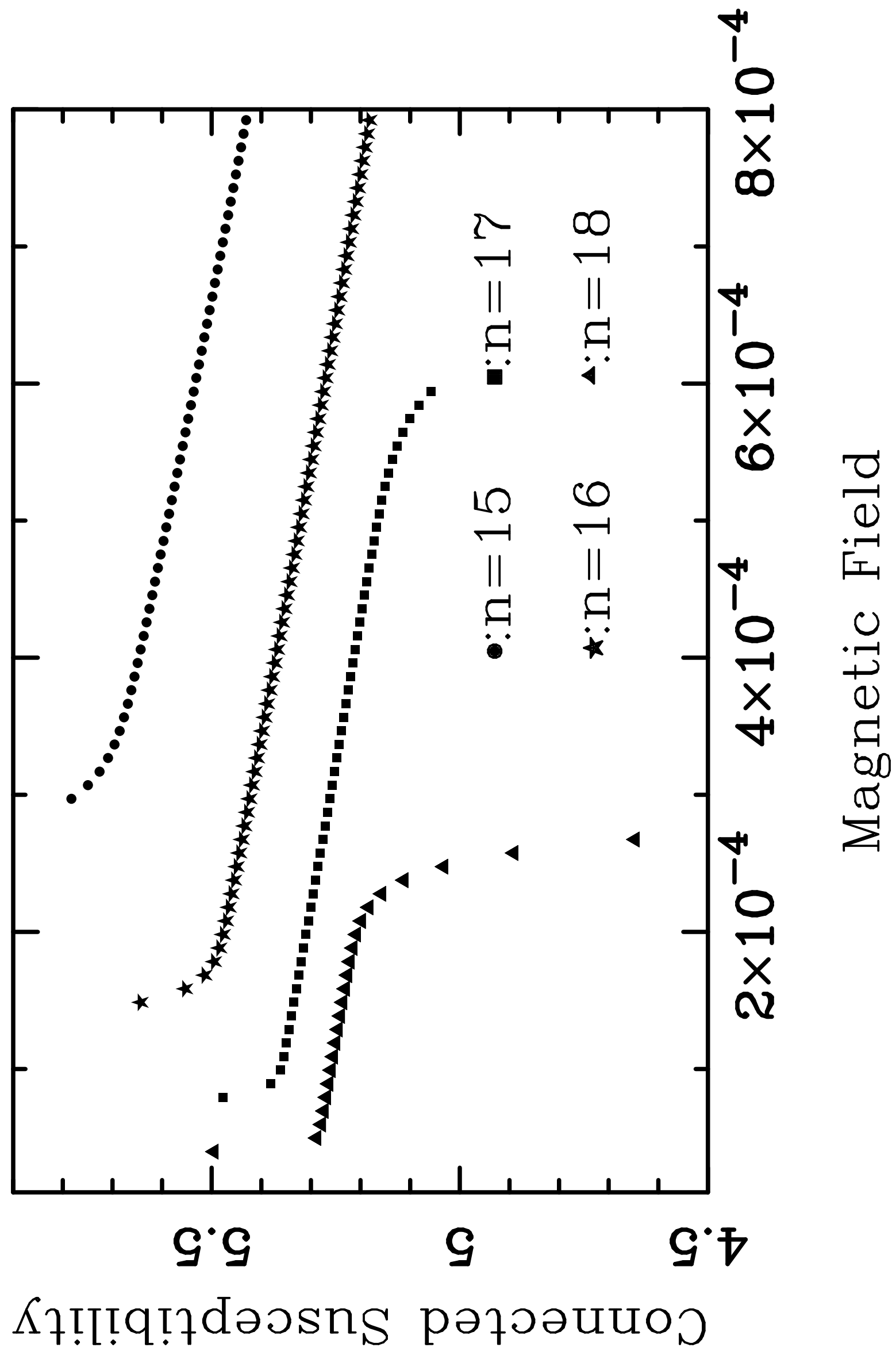

